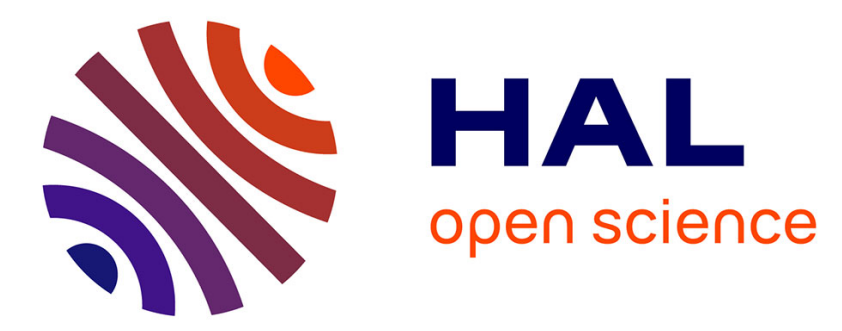

\title{
Investigation of an impinging heated jet for a small nozzle-to-plate distance and high Reynolds number: An extensive experimental approach
}

Pierre Grenson, Olivier Léon, Philippe Reulet, Bertrand Aupoix

\section{To cite this version:}

Pierre Grenson, Olivier Léon, Philippe Reulet, Bertrand Aupoix. Investigation of an impinging heated jet for a small nozzle-to-plate distance and high Reynolds number: An extensive experimental approach. International Journal of Heat and Mass Transfer, 2016, 102, p. 801 - 815. 10.1016/j.ijheatmasstransfer.2016.06.076 . hal-01393401

\author{
HAL Id: hal-01393401 \\ https://hal.science/hal-01393401
}

Submitted on 7 Nov 2016

HAL is a multi-disciplinary open access archive for the deposit and dissemination of scientific research documents, whether they are published or not. The documents may come from teaching and research institutions in France or abroad, or from public or private research centers.
L'archive ouverte pluridisciplinaire HAL, est destinée au dépôt et à la diffusion de documents scientifiques de niveau recherche, publiés ou non, émanant des établissements d'enseignement et de recherche français ou étrangers, des laboratoires publics ou privés. 


\title{
Investigation of an impinging heated jet for a small nozzle-to-plate distance and high Reynolds number: An extensive experimental approach
}

\author{
Pierre Grenson ${ }^{\mathrm{a}}$, Olivier Léon ${ }^{\mathrm{a}}$, Philippe Reulet ${ }^{\mathrm{a}}$, Bertrand Aupoix $^{\mathrm{a}}$ \\ ${ }^{a}$ DMAE, UFT-MiP, Onera, The French Aerospace Lab, 31000 Toulouse, France
}

\begin{abstract}
The present work aims at investigating a particular impinging jet configuration throughout a comprehensive experimental approach. A preheated air jet at $130^{\circ} \mathrm{C}$ issues a fully developed circular pipe at Reynolds number 60,000 and discharges in the laboratory room to impinge a flat plate located 3 diameters downstream. The description of the velocity field and the complete Reynolds stress tensor is provided by stereoscopic particle image velocimetry (S-PIV) and laser Doppler velocimetry (LDV) measurements. For the first time, data are reported for the mean and fluctuating temperature of an impinging jet configuration with the help of cold-wire thermometry (CWT) measurements. The heat transfer distribution on the impinged plate is determined through an inverse method based on infrared thermography measurements on the rear face of the plate. The agreement between S-PIV and LDV measurements is shown excellent over the whole flow field. The measured Nusselt number distribution exhibits a secondary maximum at $r / D=2$, as observed in previous experiments for short impinging distances. Flow dynamics is characterized through a spectral analysis of time-resolved measurements while flow topology features are identified through coherent structure detection based on S-PIV spatiallyresolved instantaneous velocity fields. This analysis shows that the jet column mode, associated with a Strouhal number of 0.4 , plays a key role in the primary structure dynamics.
\end{abstract}

Keywords: jet impingement, heat transfer, velocity field, temperature field, turbulent flow, primary structure dynamics, spectra

\section{Nomenclature}

$\mathrm{Bi}$

$C_{p} \quad$ plate specific heat capacity $\left(\mathrm{kgm}^{2} \mathrm{~s}^{-2} \mathrm{~K}^{-1}\right)$

$D \quad$ pipe diameter $(\mathrm{m})$

$e \quad$ plate thickness $(\mathrm{m})$

Fo Fourier number $(-)$

$h \quad$ convective heat transfer coefficient $\left(\mathrm{Wm}^{-2} \mathrm{~K}^{-1}\right)$

$H$ impingement height (m)

$k_{f} \quad$ fluid thermal conductivity $\left(\mathrm{Wm}^{-1} \mathrm{~K}^{-1}\right)$

$k_{p} \quad$ plate thermal conductivity $\left(\mathrm{Wm}^{-1} \mathrm{~K}^{-1}\right)$

$L \quad$ impinged plate length (m)

$\mathrm{Nu}_{D}$ diameter-based Nusselt number $(-)$

$\mathrm{Nu}_{L} \quad$ plate length-based Nusselt number (-)

$P \quad$ frequency-peak emergence criterion $(-)$

$q_{c} \quad$ convective heat transfer $\left(\mathrm{Wm}^{-2}\right)$

$R \quad$ pipe radius $(\mathrm{m})$

$\mathrm{Ra}_{L} \quad$ plate length-based Rayleigh number (-)

$\operatorname{Re}_{D}$ diameter-based Reynolds number (-)

$\operatorname{Re}_{\tau} \quad$ pipe friction Reynolds number (-)

$\mathrm{St}_{D} \quad$ diameter-based Strouhal number $(-)$

$t \quad$ time (s)

$T$ temperature $\left({ }^{\circ} \mathrm{C}\right)$

$T_{f} \quad$ film temperature $\left({ }^{\circ} \mathrm{C}\right)$

Email address: pierre.grenson@onera.fr (Pierre Grenson)
$T_{\text {ref. }} \quad$ reference temperature $\left({ }^{\circ} \mathrm{C}\right)$

$T_{a w} \quad$ adiabatic wall temperature $\left({ }^{\circ} \mathrm{C}\right)$

$T_{e} \quad$ ambient temperature $\left({ }^{\circ} \mathrm{C}\right)$

$T_{j} \quad$ jet exit temperature $\left({ }^{\circ} \mathrm{C}\right)$

$T_{w} \quad$ wall temperature $\left({ }^{\circ} \mathrm{C}\right)$

$T_{w}^{\text {rear }}$ rear-face wall temperature $\left({ }^{\circ} \mathrm{C}\right)$

$u_{x}, u_{r}, u_{\theta}$ cylindrical velocity components $\left(\mathrm{ms}^{-1}\right)$

$u_{x}, u_{y}, u_{z}$ cartesian velocity components $\left(\mathrm{ms}^{-1}\right)$

$u_{\tau} \quad$ friction velocity $\left(\mathrm{ms}^{-1}\right)$

$U_{j} \quad$ jet bulk velocity $\left(\mathrm{ms}^{-1}\right)$

$U_{m} \quad$ jet centerline velocity $\left(\mathrm{ms}^{-1}\right)$

$x, r, \theta$ cylindrical coordinates $(\mathrm{m}, \mathrm{m}, \mathrm{rad})$

$x, y, z$ cartesian coordinates $(\mathrm{m}, \mathrm{m}, \mathrm{m})$

Greek symbols

$\alpha \quad$ plate thermal diffusivity $\left(\mathrm{m}^{2} \mathrm{~s}^{-1}\right)$

$\beta \quad$ coefficient of thermal expansion $\left(\mathrm{K}^{-1}\right)$

$\Delta t \quad$ time step (s)

$\Delta T \quad$ jet to ambiant temperature difference $\left({ }^{\circ} \mathrm{C}\right)$

$\Delta x \quad$ spatial discretization in plate thickness $(\mathrm{m})$

$\eta \quad$ effectiveness $(-)$

$\nu \quad$ kinematic viscosity $\left(\mathrm{m}^{2} \mathrm{~s}^{-1}\right)$

$\rho_{p} \quad$ impinged plate density $\left(\mathrm{kgm}^{-3}\right)$

Other symbols

\langle\rangle$\quad$ ensemble-averaged quantity

fluctuating part

RMS root-mean-square 


\section{Introduction}

During the last 50 years, the impinging jet configuration has received considerable attention for two main reasons. Firstly, the high heat transfer rate experienced by the targeted surface explains its widespread use in industrial applications where thermal efficiency is of prior interest, such as in turbine blade cooling or aircraft leading edge heating for anti-icing purpose. Secondly, the flow characteristics of impinging jets have been proved complex to predict through numerical simulation (Zuckerman and Lior, 2006; Dewan et al., 2012) despite the geometric simplicity of the configuration. Hence, the impinging jet configuration is still a reference test case for turbulence modeling. In this context, the validation process requires accurate and welldocumented experimental databases providing complete information about the flow field and heat transfer rates. The aim of the present study is to provide a joint description of both the velocity and temperature fields along with the heat transfer coefficient distribution of a little-studied impinging jet configuration. The experimental data provide both mean and fluctuating fields together with the flow topology and a spectral characterization.

\subsection{State-of-the-art}

The major reviews related to jet impingement are provided by Martin (1977), Jambunathan et al. (1992), Viskanta (1993) and more recently Carlomagno and Ianiro (2014). From these reviews it was shown that the flow characteristics and the heat transfer distribution on the impinged plate are influenced by various parameters such as the jet Reynolds number, the impingement height, the confinement, the nozzle shape or the turbulence intensity at the nozzle exit. The impinging-jet flow is generally split into three zones that feature different characteristic behaviors : the free jet, the stagnation and the wall jet regions (Fig. 1).

The early contributions of Gardon and Akfirat (1965) and Hoogendoorn (1977) evidenced the importance of the jet turbulence in heat transfer processes. The jet turbulence principally depends on the jet exit conditions. As jet exit, most studies used either convergent nozzles, for which turbulence is only confined in thin boundary layers, or fully developed pipe flow. The dependence of the heat transfer on the Reynolds number $\operatorname{Re}_{D}$ and the nozzle-to-plate spacing $H / D$ was thoroughly investigated through the experiments Lytle and Webb (1994); Hofmann et al. (2007); Katti and Prabhu (2008); Lee and Lee (1999) that followed the pioneering work of Baughn and Shimizu (1989). The considered jet Reynolds number range was $5,000<\operatorname{Re}_{D}<150,000$ and the nozzle-to-plate spacing $H / D$ varied between 0.25 and 10 . The heat transfer coefficient was shown to present a maximum at the stagnation point, the level of which depends on $H / D$. The optimal heat transfer coefficient was reached for $H / D=6$, which appears to coincide with the potential core length

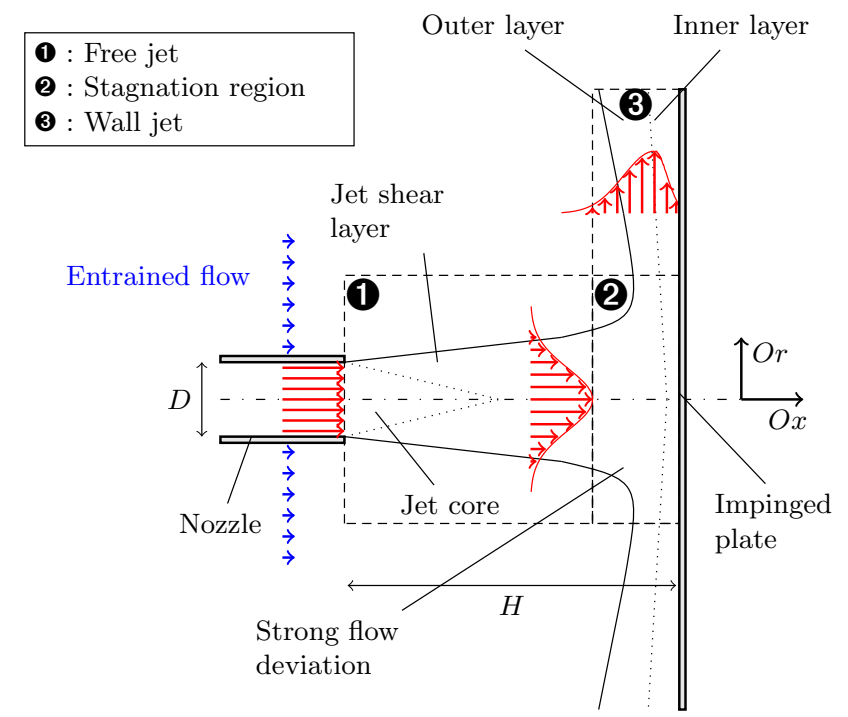

Figure 1: Schematic description of a round jet impinging on a flat plate.

of the jet (Gardon and Akfirat, 1965). For low nozzle-toplate distances $H / D<4$, the radial distribution of heat transfer coefficient was shown to exhibit a secondary peak (Baughn and Shimizu, 1989). Its location, which varies from $r / D=1.2$ to $r / D=2.1$, and intensity both depend on the Reynolds number and the nozzle-to-plate distance. Several explanations for its appearance were proposed. The today most accepted interpretation relates the secondary maximum to the interaction of primary vortices, originated from the jet shear layer, with the impingement plate. This hypothesis is supported by the recent numerical simulations of Hadžiabdić and Hanjalić (2008) and Dairay et al. (2015) and the experiments of O'Donovan and Murray (2007) through simultaneous acquisition of heat transfer and velocity.

The mean and fluctuating properties of the flow field were first addressed by (Cooper et al., 1993) through hotwire anemometry measurements (HWA) for a jet which issues a fully developed long pipe at $\operatorname{Re}_{D}=23,000$ and 70,000 , for $H / D$ ranging from 2 to 10 . The experiments of Nishino et al. (1996), which were based on particle tracking velocimetry, provided important information about turbulence statistics in the stagnation region such as regions of negative production of turbulent kinetic energy. Guerra et al. (2005) performed both HWA and thermocouple measurements of the mean temperature over a heated plate for $H / D=2$ and $\operatorname{Re}_{D}=35,000$. Geers (2004) carried out both LDV and PIV measurements on Cooper's configuration. This configuration was again investigated by Tummers et al. (2011) through LDV measurements which were specifically tuned for near-wall exploration.

In the last decade, the improvement in the PIV capabilities led to numerous investigations which focused on the impinging jet flow topology (Hammad and Milanovic, 2011; Roux et al., 2011). Time-resolved tomographic PIV was applied on low-Reynolds number $\left(\operatorname{Re}_{D}<5,000\right)$ im- 
pinging jet at short impinging distances by Violato et al. (2012) and Sodjavi et al. (2016). These studies revealed the three dimensional large scale turbulent structures generated in the free jet and their subsequent interaction with the plate, leading to creation of counter-rotating secondary vortices in the near wall region. This interaction, often referred to as unsteady separation, was first introduced by Didden and Ho (1985) and observed on the early smoke visualizations of Popiel and Trass (1991) and the PIV of Landreth and Adrian (1990) for low-Reynolds number jets out of a convergent nozzle. The same phenomenon was retrieved for higher Reynolds numbers, both experimentally (Tummers et al., 2011; Meola et al., 2000) and numerically (Hadžiabdić and Hanjalić, 2008; Dairay et al., 2015).

The aforementioned works primarily concerned isothermal jets, i.e the jet temperature $T_{j}$ is equal to the ambient temperature $T_{e}$, the plate being heated. Several studies (Striegl and Diller, 1984; Goldstein et al., 1990; Baughn et al., 1991; Brevet, 2001; Vinze et al., 2016) were dedicated to the jet temperature effects. Their major outcome, for 5,000 $<\operatorname{Re}_{D}<121,000$ and $0<\Delta T<150^{\circ} \mathrm{C}$, was that, on a given configuration, the Nusselt number distribution for heated and unheated jet collapses on the same curve, provided that the adiabatic wall temperature is taken as reference temperature in the local convective heat transfer coefficient. In addition, the effectiveness (the definition of which is reminded in AppendixA) was proved very sensitive to the nozzle-to-plate distance while being almost independent of both the jet Reynolds number and the temperature difference between the jet flow and the surroundings $\Delta T=T_{j}-T_{e}$.

\subsection{Scope of the present work}

Notwithstanding the numerous works dedicated to the impinging jet configuration, several authors (Behnia et al., 1999; Brevet, 2001) pointed out the results scattering between experiments due to the various factors influencing the flow and the lack of information about the setup parameters, such as the jet exit condition (velocity profiles and turbulence level). The difference in the measurements techniques can also explain the mismatch in the results obtained on similar configurations. Moreover, experimental studies addressing at the same time the flow dynamics together with the heat transfer distribution are rather scarce. In the framework of numerical simulation validation, this leads to a combination of different data sets, that indubitably introduces undetermined biases irrespective of the simulation ability to predict the flow field and heat transfer.

For these reasons, the present study aims at providing a well documented experimental database on a selected jet impingement configuration. The state-of-the-art revealed that the configuration $\operatorname{Re}_{D} \simeq 20000$ and $H / D=2$ was the most frequently chosen one in order to facilitate the comparison with the early experiments of Cooper et al. (1993) and Baughn and Shimizu (1989). However, the increase in computer power nowadays allows for unsteady numerical simulation of jets at higher Reynolds number. In addition, description of flow topology and dynamics at Reynolds number higher than $\operatorname{Re}_{D}=30,000$ has never been reported. For the current investigation, we therefore retained a higher Reynolds number of $\operatorname{Re}_{D}=60,000$. A nozzle-to-plate distance of $H / D=3$ was selected after preliminary measurements of the Nusselt number distribution for different ratios $H / D$ between 1 and 8 . This choice of small nozzle-to-plate spacing gives the ability to perform direct or large-eddy simulations of the configuration with reasonable mesh size. For the jet exit condition, we selected the fully developed pipe condition for its genericity: the velocity profile and the turbulence at the pipe exit are uniquely defined for a given Reynolds number. The air jet is heated in order to get a sufficient temperature difference with the surroundings, providing the opportunity to perform measurement of the thermal field, which has never been carried out on an impinging jet configuration (to the authors' knowledge).

One interest of the present work lies in the various experimental techniques which were employed. The velocity field was measured using two different techniques: laser Doppler velocimetry (LDV) and stereoscopic particle image velocimetry (S-PIV). On the one hand, this redundancy allowed to assess the reliability of the presented results. On the other hand, the combination of time-resolved measurements yielded by LDV with instantaneous vector fields obtained by S-PIV allowed for a better understanding of the flow dynamics. The use of a cold wire thermometer (CWT) gave access to both the mean and fluctuating temperature fields along with temperature spectra. Finally, both the heat transfer and the adiabatic wall temperature distribution on the impinged plate were obtained by means of the inverse method (ThEFA) proposed by Reulet et al. (2003), which is based on infrared thermography measurements of the rear face of the plate. Contrary to the thin foil technique, this method offers the advantage that the plate does not have to be supplied by a constant heat flux but is simply introduced in the hot flow.

The paper is organized as follows. Sec. 2 describes the test rig (Sec. 2.1) and the relevant parameters of the measurement techniques (Sec. 2.2). Sec. 3 presents the main results. The fully developed state of the flow at the pipe exit is first assessed in Sec. 3.1. Sec. 3.2 describes the temperature distribution on the plate at equilibrium condition. Next, the mean and fluctuating velocity and temperature fields are characterized in Sec. 3.3 with a particular attention paid on velocity and temperature profiles above the plate. Quality of the measurements is also discussed. In Sec. 3.4, the heat transfer coefficient and the adiabatic wall temperature distribution as measured by ThEFA are compared to previous experimental results from literature. In Sec. 3.5, a vortex detection algorithm is applied to the S-PIV instantaneous velocity measurements in order to locate coherent structures. Afterwards, the spectra from the time-resolved measurements are dealt with in Sec. 3.6. Finally, Sec. 3.7 relates the presence of coherent structures 
above the plate to the emergence of a characteristic frequency in this region of the flow.

\section{Experimental Setup}

\subsection{Test rig}

The experimental setup is sketched in Fig. 2. It consists of an insulated long pipe and the impinged plate. The origin of the orthogonal coordinate system is located at the pipe outlet center. The jet axis is along the $x$ direction and is taken as positive in the jet mean direction while $y$ and $z$ axes lie in the exit plane.

The continuous air flow entering the pipe is provided by a compressor and heated by an electrical heat exchanger. Both air mass flow rate and pipe inlet temperature are regulated. Measurements are performed at a mass flow rate of $63.5 \mathrm{gs}^{-1}$, which corresponds to a bulk velocity of $U_{j}=25.7 \mathrm{~ms}^{-1}$. The Reynolds number based on the exit bulk velocity is $R e_{D}=U_{j} D / \nu=60,000$. The pipe has an inner diameter $D=60 \mathrm{~mm}$ and its length is $50 D$, which is required to generate a fully developed flow at the considered Reynolds number (Zagarola and Smits, 1998). A rectifier is located at the pipe entrance in order to avoid swirling effects. Regulated heating collars, wrapped around the pipe, maintain the flow temperature at $T_{j}=130^{\circ} \mathrm{C}$. An outer cylindrical case of diameter $270 \mathrm{~mm}$, encircling the pipe, is filled with an insulant material (cellulose wadding) to minimize heat losses. A perforated circular teflon ring of inner diameter $D$ is placed at the outlet section of the pipe between the outer cover and the pipe. Its external diameter with respect to the impingement height leads to a semi-confined configuration. The hot jet finally discharges into the laboratory room maintained at an ambient temperature $T_{e}=25^{\circ} \mathrm{C}$.

The $3 \mathrm{~mm}$-thick impacted square plate is made of titanium T40 (Table A.3). It is mounted orthogonally to the jet axis on an independent carriage which gives the ability to vary the impingement distance. In the present study, the exit-to-plate distance is fixed at $H / D=3$. Two impinging plates are used depending on the measurement technique. For flow field measurements, the plate is $600 \mathrm{~mm} \times 600 \mathrm{~mm}$ and fixed at its four corners. For the wall heat transfer characterization, the plate $(400 \mathrm{~mm} \times 400 \mathrm{~mm})$ is embedded in a larger frame attached to a lateral moving carriage ( $z$-direction) giving the ability to quickly insert the plate into the jet flow.

The thermal environment of the experimental setup is monitored by $K$-type thermocouples (TC). The reference jet temperature $T_{j}$ is measured near the inlet of the pipe while the surrounding conditions are evaluated by 2 TCs respectively positioned in the neighborhood of the outer case and behind the impinged plate. Several TCs are placed around the inner pipe to control that pipe temperature stays close to the operating conditions. The quality of the regulation ensures that the variations of the reference temperature do not exceed $\pm 0.5{ }^{\circ} \mathrm{C}$.

\subsection{Measurement techniques}

The experimental arrangements associated with the different measurement techniques are presented in Fig. 3. Table 1 sums up their major features and outcome.

Table 1: Measurement methods. SR states for spatially resolved measurement and TR for time-resolved measurement with the highest allowable frequency specified.

\begin{tabular}{|c|c|c|c|}
\hline Technique & Resolution & Quantity & Localization \\
\hline \multirow{2}{*}{ LDV } & $\mathrm{TR}$ & $u_{x}$ & 1500 in flow \\
\hline & $\sim 10 \mathrm{kHz}$ & $u_{z}$ & points \\
\hline \multirow{3}{*}{ S-PIV } & \multirow{3}{*}{ SR } & $\overline{u_{x}}$ & 9 observation \\
\hline & & $u_{y}$ & windows in the \\
\hline & & $u_{z}$ & $O x y$-plane \\
\hline CWT & $\begin{array}{c}\mathrm{TR} \\
6 \mathrm{kHz}\end{array}$ & $T$ & $\begin{array}{l}1500 \text { in flow } \\
\text { points }\end{array}$ \\
\hline ThEFA & SR & $\begin{array}{c}T_{w} \\
\mathrm{Nu}_{D} \\
T_{a w}\end{array}$ & impinged plate \\
\hline
\end{tabular}

\subsubsection{Laser Doppler Velocimetry (LDV)}

The LDV velocimeter (Fig. 3a) consists of a $1 \mathrm{~W}$ Spectra-Physics argon ion laser emitting a $514 \mathrm{~nm}$ (green) and $476 \mathrm{~nm}$ (blue) wavelength pair of beams through a TSI-9832 emitting head, which enables to measure two velocity components. The receiving head, linked to an IFA755 burst spectrum analyzer, is set up in the forward configuration, which maximizes the light scattered by the oil particles. The measurement volumes are about $0.10 \mathrm{~mm}$ in diameter and $1.6 \mathrm{~mm}$ long, with the long axis aligned in the $z$ direction. Atomized DEHS oil, yielding droplets less than $1 \mu \mathrm{m}$ in diameter, is used as tracer. The flow issuing the pipe is seeded through a thin tube discharging in the pipe as shown in Fig. 2. A set of tubes is placed around the pipe outer carter, upstream of the exit plane, in order to seed the surrounding air. Velocity weighting and statistics are performed using the ONERA in-house software ASSA (Micheli et al., 2006) which is specifically dedicated to multichannel random-sampled signals.

\subsubsection{Stereoscopic Particle Image Velocimetry (S-PIV)}

The S-PIV system (Fig. 3b) is composed of two CCD 12-bits IMAGER-SX cameras of 2,330 px × 1,777 px resolution and a double-pulsed Nd:YAG Quantel laser of $200 \mathrm{~mJ}$ energy per pulse at a wavelength of $532 \mathrm{~nm}$. Synchronization is carried out by a LaVision Controller and the data acquisition is performed with DaVis 8.2 software. The $1 \mathrm{~mm}$-thick light sheet lies in the $O x y$ plane containing the jet axis. The cameras are located on each side of the sheet in a forward-forward arrangement optimizing the Mie scattering. The angle between the two cameras and the laser sheet is $45^{\circ}$ which allows for a good stereoscopic reconstruction accuracy. Both cameras are equipped with Nikkor $50 \mathrm{~mm}$ lenses along with 


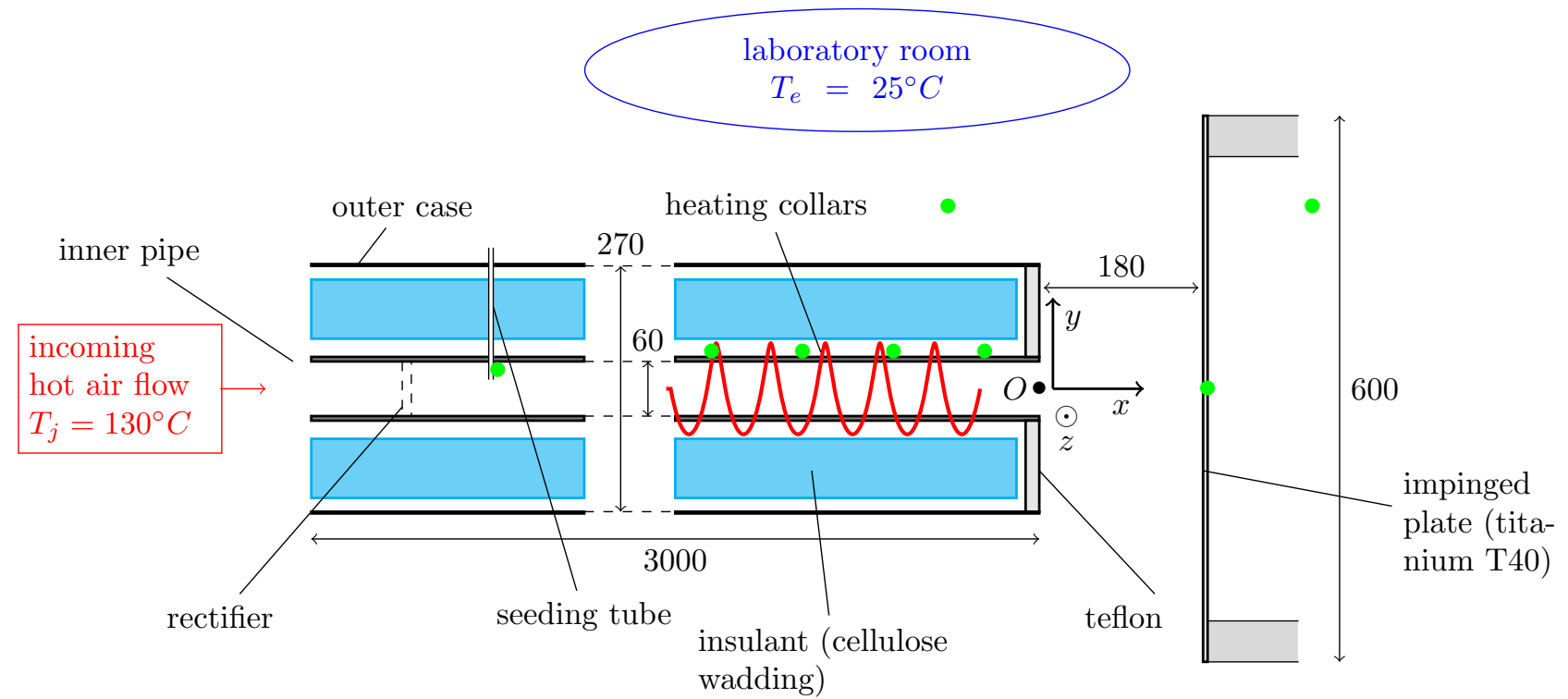

Figure 2: Experimental setup. Green dots $(\bullet)$ show the TC locations. Dimensions are in $\mathrm{mm}$.

Scheimpflug adapters which enable to keep the best focus quality over the whole field of view. To limit laser reflection seen by the cameras, the impinged plate and the exit teflon surface are coated with Rhodamine 6D and a narrow bandpass filter is mounted on the cameras. The seeding of both pipe and quiescent flows is identical to that of the LDV measurements, described above. The domain of interest is divided in 9 measurement windows, each of them corresponding to a physical surface of $100 \mathrm{~mm} \times 90 \mathrm{~mm}$. A number of 2000 independent image pairs per camera is recorded at a sampling rate of $10 \mathrm{~Hz}$ for each measurement window. In order to process each image pairs and compute the three-components vector field, the in-house software FOLKI-SPIV (Champagnat et al., 2011) has been selected owing to its efficiency (calculations are performed on GPUs) and accuracy. The algorithm, which is based on the Lucas-Kanade paradigm, performs a moving average the width of which is driven by the size of the interrogation windows. Considering the good seeding density, a window width of $15 \mathrm{px} \times 15 \mathrm{px}$ is selected, leading to a spatial resolution of the computed velocity field of $0.87 \mathrm{~mm}$.

\subsubsection{Cold Wire Thermometry (CWT)}

The temperature measurements (Fig. 3c) are performed with a DANTEC 55P31 cold wire probe linked to the constant-current thermometry module DANTEC 90C20. The probe is mounted on a 3-axis traverse system. Its support is adapted depending on the investigated domain (free jet: configuration 1 or wall jet zone: configuration 2 ) in order to minimize the undesirable intrusive effects. The sampling rate is $6 \mathrm{kHz}$ and the signal duration for each measured single-point is $30 \mathrm{~s}$. Calibration of the linear voltage-temperature law is carried out in situ with a Pt100 thermometer certified by a COFRAC calibration at $\pm 0.1^{\circ} \mathrm{C}$.

\subsubsection{Infrared Thermography and ThEFA method}

Fig. 3d shows the experimental arrangement associated with the heat transfer coefficient measurement. The local convective heat flux coefficient and the adiabatic temperature on the impinged plate are obtained from the local transient evolution of the rear surface temperature when the plate is suddenly introduced in the hot jet flow, following the ThEFA (French acronym for rear face thermography) method introduced by Reulet et al. (2003) and presented in AppendixA.

The Biot number $\mathrm{Bi}=h e / k_{p}$ calculated from titanium plate thermal conductivity $k_{p}$ and thickness $e$ along with the convective heat flux coefficient $h$ is $\sim 0.02<0.1$, which means that the plate is thermally thin. Besides, the thermal diffusion time $t_{\alpha}=e^{2} / \alpha$ in the thickness direction is $1.1 \mathrm{~s}$, which is low compared to the transient duration of $180 \mathrm{~s}$. The front face temperature $T_{w}$ is therefore close to the rear face temperature $T_{w}^{\text {rear }}$ during the plate-heating transient.

The temperature measurements are carried out with a FLIR SC7650 MWIR camera (640 px $\times 512$ px resolution), mounted with a $25 \mathrm{~mm}$ lens. The rear face of the plate is covered by several coats of paint with a known high emissivity. The uncertainty on the temperature measurement is estimated at about $\pm 3{ }^{\circ} \mathrm{C}$. Due to space constraints, a gold-coated mirror with $45^{\circ}$-angle with respect to the plate deviates the infrared radiation to the camera located $2 \mathrm{~m}$ away from the mirror. The spatial resolution is $1.3 \mathrm{~mm} / \mathrm{px}$.

\section{Results and Discussion}

Because of the statistical axisymmetry of the round jet configuration, the velocity vector components are classically described in the cylindrical reference frame i.e axial $u_{x}$, radial $u_{r}$ and azimuthal $u_{\theta}$ velocity components. The 


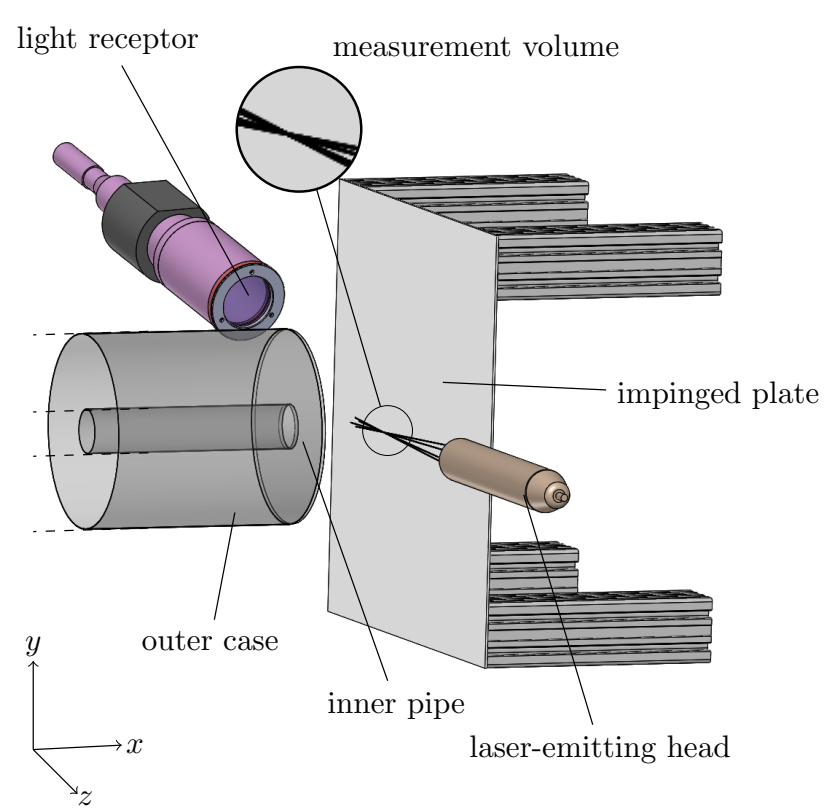

(a) LDV

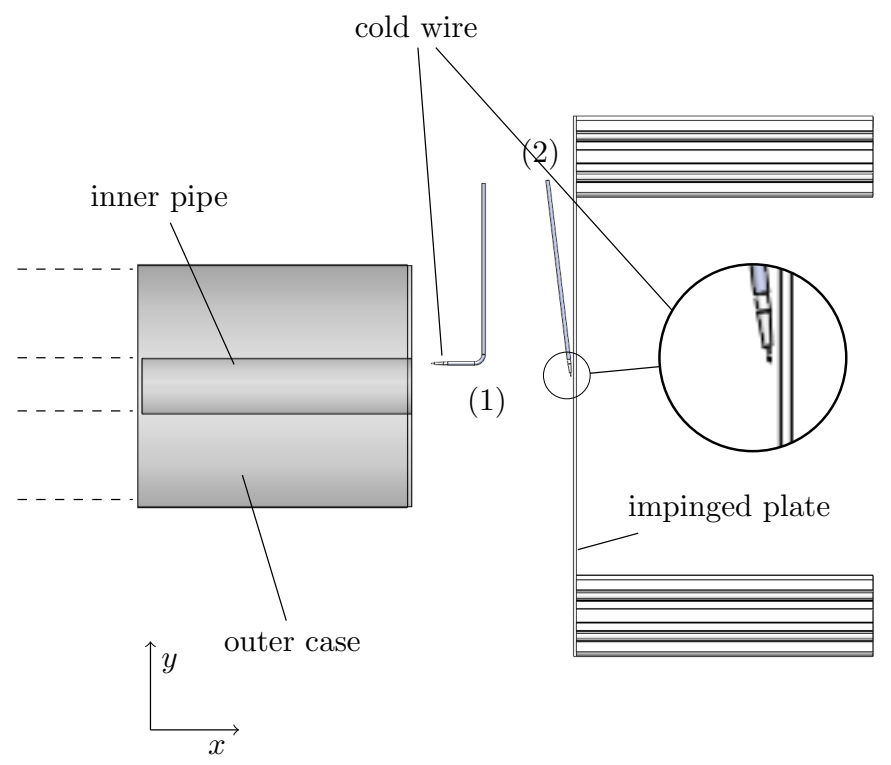

(c) $\mathrm{CWT}$

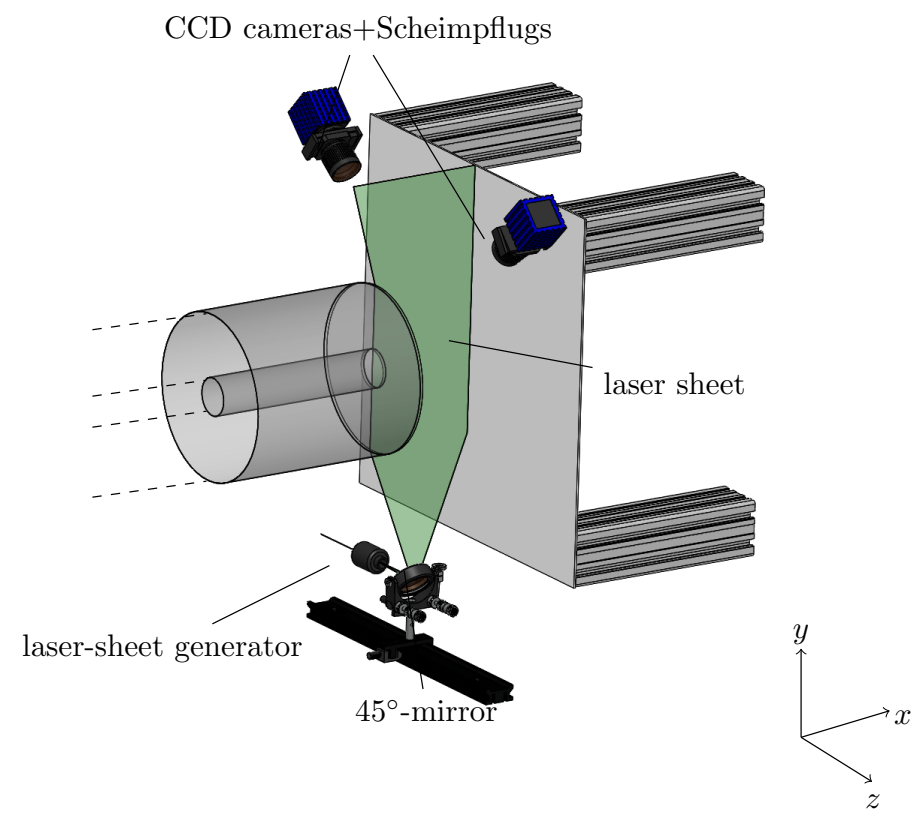

(b) S-PIV

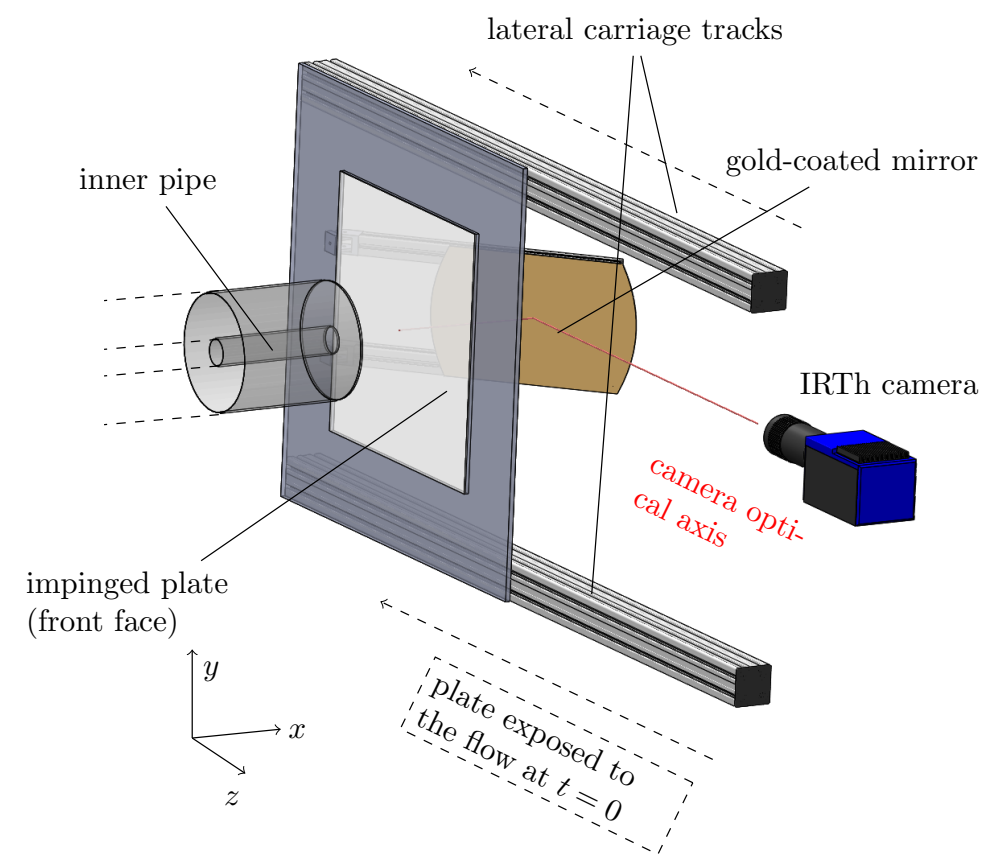

(d) Infrared Thermography (IRTh)+ThEFA

Figure 3: Measurement technique arrangements. 
actual flow symmetry with respect to the jet axis was verified by measurements in planes orthogonal to the jet axis.

\subsection{Pipe exit conditions}

The radial profiles of velocity and temperature from LDV, S-PIV and CWT measurements at axial locations close to the pipe exit are reported in Fig. 4.

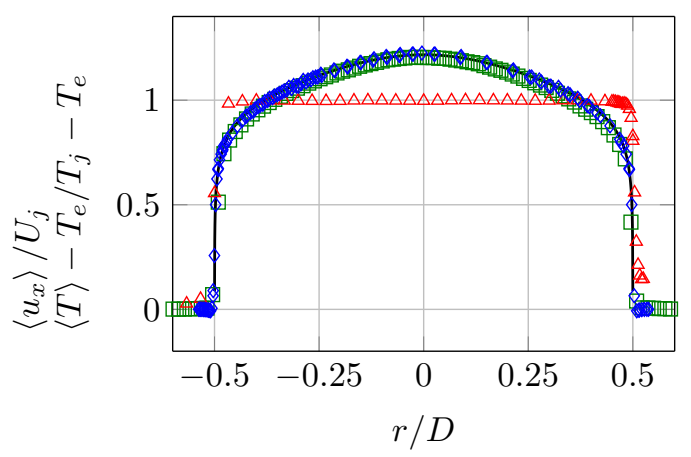

(a) Mean axial velocity and temperature.

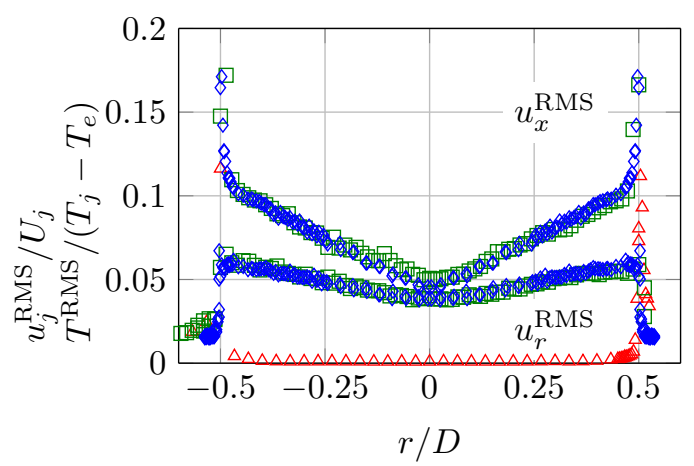

(b) Fluctuations.

Figure 4: Mean (a) and fluctuating (b) velocity and temperature profiles at the pipe exit. LDV measurements at $x / D=0.02$, SPIV at $x / D=0.1$ and CWT at $x / D=0.02$. ( $\square)$ S-PIV, $(\diamond)$ LDV, $(\Delta)$ CWT, ( - ) composite law.

In Fig. 4a, it is shown that the mean axial velocity $\left\langle u_{x}\right\rangle$ as measured by both S-PIV (green squares) and LDV (blue diamonds) is in excellent agreement with the composite law (black solid line)

$$
\frac{\left\langle u_{x}(r)\right\rangle}{u_{\tau}}=\frac{1}{\kappa} \log \left[\frac{u_{\tau}}{\nu}(r-R)\right]+C+\frac{2 \Pi}{\kappa} \mathcal{W}\left(\frac{r}{R}\right)
$$

made up of

- the $\log$-law $(\kappa=0.41$ and $C=5.0)$;

- an empirical law $\mathcal{W}(\bar{r})(\Pi=0.21)$ for the wake representation, based on the experiments of (Nagib and Chauhan, 2008) on fully developed pipes:

$$
\begin{aligned}
\mathcal{W}(\bar{r}) & =\left(1-\frac{\log (\bar{r})}{2 \Pi}\right) \times \\
& \frac{1-\exp \left(\bar{r}^{3}\left[p_{2}(\bar{r}-4 / 3)+p_{3}\left(\bar{r}^{2}-1\right)+p_{4}\left(\bar{r}^{4}-7 / 3\right)\right]\right)}{1-\exp \left[-\left(p_{2}+3 p_{3}+4 p_{4}\right) / 3\right]}
\end{aligned}
$$

where $\bar{r}=r / R, p_{2}=4.075, p_{3}=-6.911$, and $p_{4}=4.876$.

The friction velocity $u_{\tau}$ is obtained by a least-square fit of equation (1) to the experimental data. It gives $u_{\tau}=$ $1.31 \mathrm{~ms}^{-1}$, corresponding to a friction Reynolds number $\operatorname{Re}_{\tau}=u_{\tau} D / \nu=3,144$. The axial velocity at the center of the jet is $U_{m}=31.3 \mathrm{~ms}^{-1}$, yielding the ratio $U_{m} / U_{j}=$ 1.22 .

Fig. $4 \mathrm{~b}$ shows profiles of fluctuating axial $u_{x}^{\mathrm{RMS}} / U_{j}$ and radial $u_{r}^{\mathrm{RMS}} / U_{j}$ velocity components at the pipe exit. They feature the phenomenology generally observed (Toonder and Nieuwstadt, 1997; Wu and Moin, 2008) in fully developed pipe flows: the axial and radial velocity fluctuations are $\sim 5 \%$ at the center of the pipe outlet while the axial velocity fluctuations reach $\sim 18 \%$ in the vicinity of the pipe wall.

As shown in Fig. 4 the mean temperature (red triangles), represented by its dimensionless expression $(\langle T\rangle-$ $\left.T_{e}\right) /\left(T_{j}-T_{e}\right)$, is uniform across the pipe section and equal to the operating condition $T_{j}=130^{\circ} \mathrm{C}$. Moreover, the temperature fluctuations are almost equal to zero (Fig. 4b). Because the temperature profile was not strictly measured at the pipe exit but $x / D=0.02$ downstream, temperature fluctuations of $\sim 10 \%$ are observed around $|r / D|=0.5$ and correspond to the beginning of the jet shear-layer development.

\subsection{Wall Temperature at Steady-state}

The flow field measurements were carried out at equilibrium condition: the thin titanium plate is submitted to the hot jet flow for a long time so that the wall temperature reaches steady state. The convective heat flux $h$ on the front face of the plate is thus balanced by

- the natural convective heat flux on the rear face of the plate;

- the radiative losses with the surroundings;

- the thermal diffusion in the plate.

Fig. 5 shows the wall temperature distribution $T_{w}(y, z)$ on the front face at steady-state condition. The highest temperature $T_{w}=113^{\circ} \mathrm{C}$ is reached at the stagnation point. It is shown that the wall temperature distribution exhibits concentric lines of iso-temperature, which confirms the axisymmetry of the mean flow with respect to the jet axis.

\subsection{Flow field}

Fig. 6 gives an overall view of mean and fluctuating flow fields as determined from the S-PIV measurements in the longitudinal Oxy plane. The three characteristic zones, namely the free jet region $(\mathbf{0})$, the stagnation region (2) and the wall jet region (3) introduced in Fig. 1 are also reminded.

The mean and fluctuating temperature at the measurements points of CWT are reported in Fig. 7.

Mean and fluctuating velocity and temperature profiles at different radial locations along the plate are plotted in 


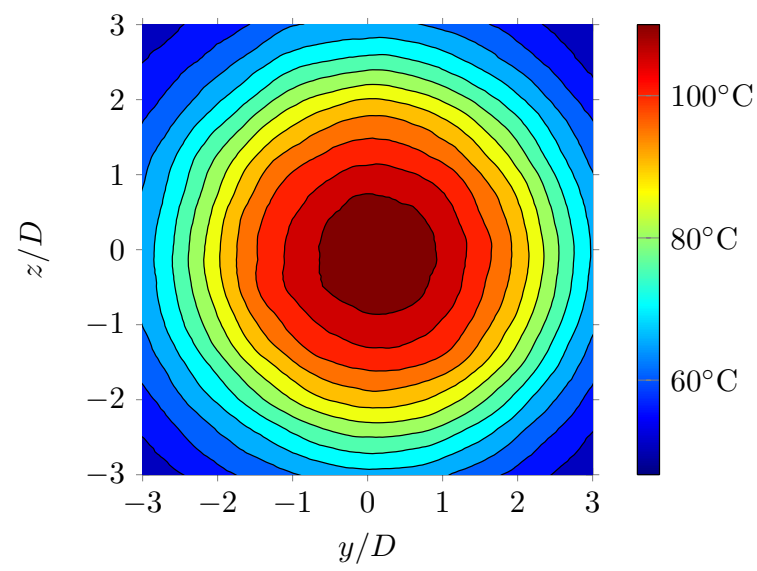

Figure 5: Wall temperature $T_{w}(y, z)$ on the plate at equilibrium condition.

Fig. 8. The wall temperature as measured by IRTh at steady-sate condition (Fig. 5) is represented in Fig. 8b by a red circle. Due to thermal effects, we observed a small concave deformation of the titanium plate as shown by the actual plate geometry represented in Fig. 6. The deformation, which does not exceed $2 \%$ of the impingement height, leads to a shift in the wall location originally located at $x / D=3$. Actual wall location is represented in Fig. 8 by a gray rectangle.

\subsubsection{Accuracy of the velocity measurements}

It can be first assessed from Figs. 6 and 7 that the symmetry with respect to the jet axis is excellent in both the free jet and the stagnation region.

Concerning the velocity profiles, a comparison between LDV and S-PIV measurements can be performed in Fig. 8. The agreement is almost perfect for the mean velocity profiles whereas only small discrepancies are observed for the fluctuating profiles. The largest differences are observed in the wall vicinity for the radial velocity fluctuations $u_{r}^{\mathrm{RMS}}$ at $r / D=1$ in Fig. 8c. It does not come as a surprise that PIV fails to provide accurate measurement in the near wall region. First, the light reflection, even though reduced by the Rhodamine coating, alters the signal to noise ratio in the neighborhood of the plate. Second, the limited spatial resolution with the present setup makes it difficult to capture strong flow variations, as encountered in this region.

The underestimation of axial velocity fluctuations $u_{x}^{\mathrm{RMS}}$ observed at $r / D=2.5$ and $r / D=3$ (Fig. 8d) with S-PIV system might be due to the lower seeding density observed far from the jet axis because of the radial expansion of the wall jet.

The mean and fluctuating radial velocity profiles at $r / D=1.5$ (Figs. 8a and 8c) are compared to the experimental data of Cooper et al. (1993), gathered on a similar jet impingement configuration $\left(\operatorname{Re}_{D}=70000\right.$ and $H / D=3$ ) by means of HWA. The excellent agreement with our velocity measurements enforces the confidence in the presented results.

\subsubsection{Mean flow}

Fig. $6 \mathrm{~b}$ shows that, after exiting the pipe, the jet flows toward the impingement plate with radial spreading (freejet region (1). When approaching the plate, the flow undergoes a strong deceleration (stagnation region (2), followed by deflection in the radial direction (Fig. 6a). The presence of the plate influences the free jet from axis location $x / D=2$. Due to the strong negative pressure gradient in the radial direction, the flow is accelerated away from the stagnation point, leading to the formation of the wall jet. The peak radial velocity reaches a maximum about $r / D=1$ (Figs. 6b and 8a). This location is often considered as the beginning of the wall jet region (3) (Jambunathan et al., 1992). The highest flow speed in the velocity profile at a given radial location decreases as the jet spreads along $r$ axis $(r / D=1$ to $r / D=3$ in Fig. 8a) because of both the radial expansion of the flow and the turbulent mixing with the low-speed ambient air. The $x / D$-location of the maximum velocity generally defines the edge between the outer layer, which exhibits features of free turbulent shear flow, and the inner layer, which is similar to a boundary layer (see vertical dashed line in Fig. 8 at $r / D=2$ ).

Figs. $7 \mathrm{a}$ and $8 \mathrm{~b}$ at $r / D=0$ show that the temperature is constant along the jet axis and equal to the jet exit temperature $T_{j}$ up to the vicinity of the wall. The stagnation point therefore lies in the core of the jet, which is defined as the internal region of the free-jet which has not yet been affected by the developing shear layers. The radial extent of the jet core diminishes at increasing axial location, as clearly visible on the mean temperature field in Fig. 7a.

The thermal wall jet evolution can be assessed in Fig. 8b, $r / D=1$ to 3 . As for the velocity profile, the maximum temperature is reached at a distance from the wall which defines the height of the inner thermal layer (see inset in Fig. $8 \mathrm{~b}, r / D=2$ ). The difference between the maximum temperature and the wall temperature (red circle in Fig. $8 \mathrm{~b})$ is rather small $\left(\sim 20^{\circ} \mathrm{C}\right)$ because the temperature measurements is carried out at the thermal equilibrium condition, as mentioned in Sec. 3.2. The wall heat flux is then low and corresponds to the heat losses listed in Sec. 3.2. When moving along the $r$ axis, the maximum temperature decreases due to the mixing with the ambient cold air in the outer layer of the wall jet, as for the velocity.

\subsubsection{Fluctuating flow}

In the free jet region $(\mathbf{(})$, the annular mixing layer grows downstream from the jet exit but does not reach the jet axis before impinging the plate, confirming that the stagnation point lies in the jet core (Figs. 6c, 6d, 6e and 7b). In this region, the velocity perturbations are mainly due to turbulence initially present at the pipe exit. The temperature fluctuations $T^{\mathrm{RMS}}$ are almost equal to zero (Fig. 8e, $r / D=0)$. In the central region of the mixing layer, the velocity fluctuations are high: axial velocity fluctuation $u_{x}^{\mathrm{RMS}}$ reaches $18 \%$ while the velocity component fluctuations are ordered classically $u_{x}^{\mathrm{RMS}}>u_{\theta}^{\mathrm{RMS}}>u_{r}^{\mathrm{RMS}}$. 


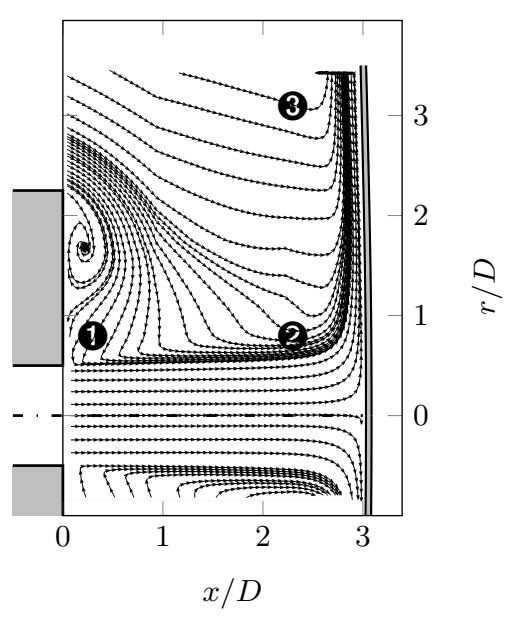

(a) Streamlines
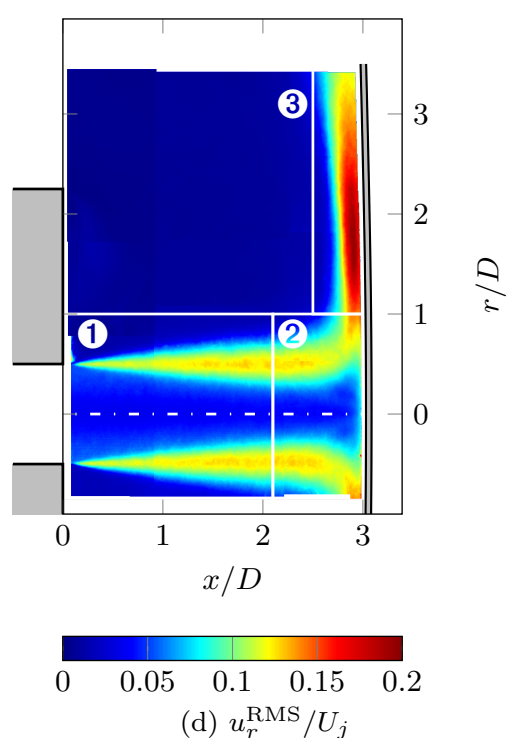
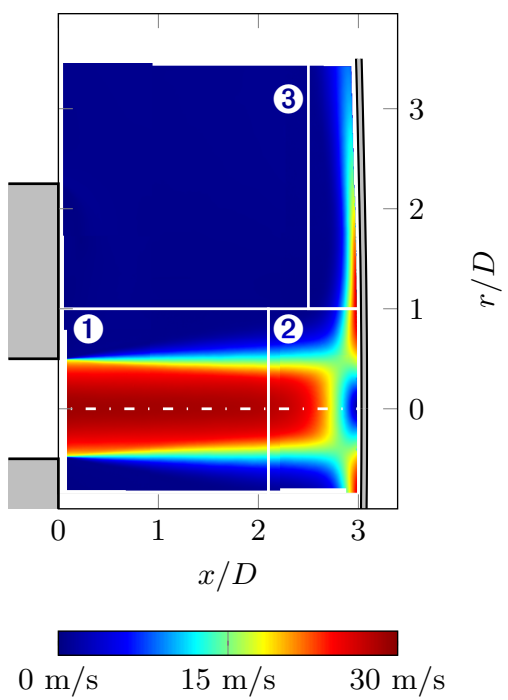

(b) $\langle u\rangle / U_{j}$
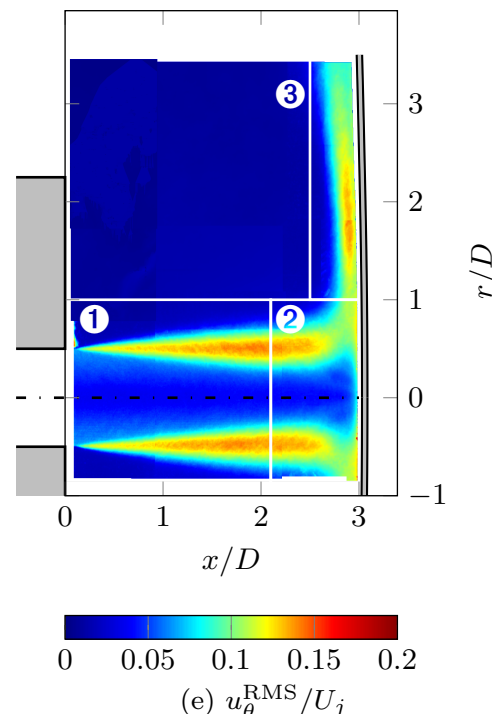
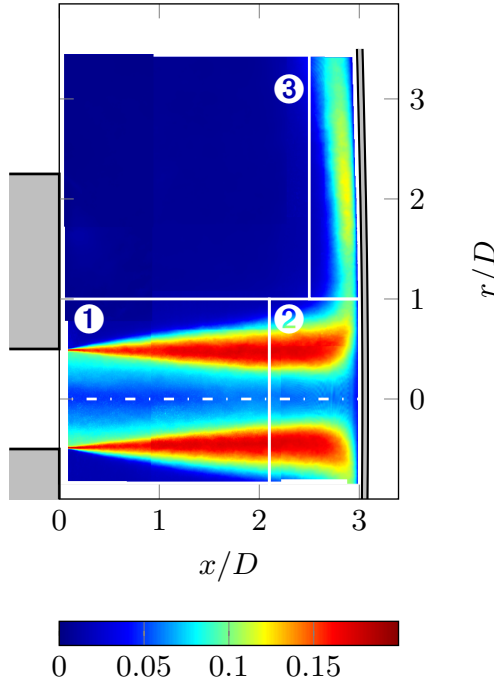

(c) $u_{x}^{\mathrm{RMS}} / U_{j}$
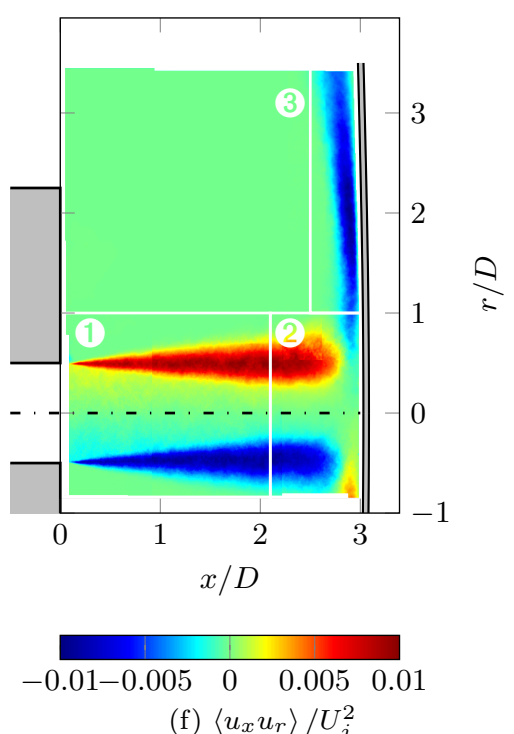

Figure 6: S-PIV statistical field. $\mathbf{0}$ : free jet region - 2 : stagnation region - 3 : wall jet region 


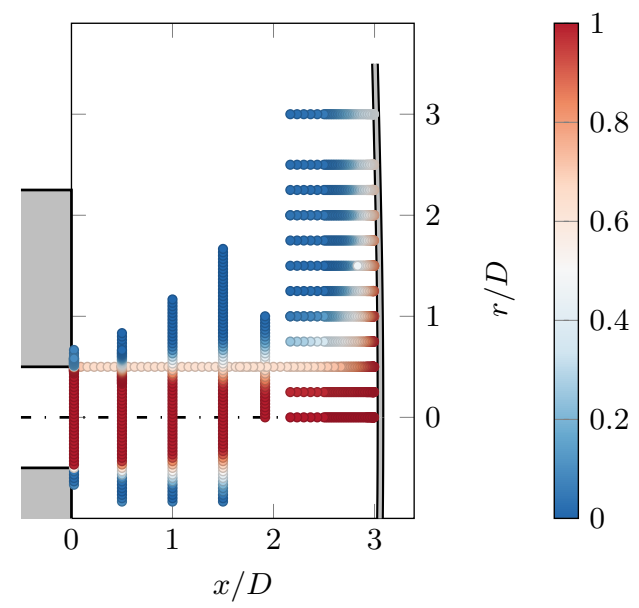

(a) $\left(\langle T\rangle-T_{e}\right) /\left(T_{j}-T_{e}\right)$

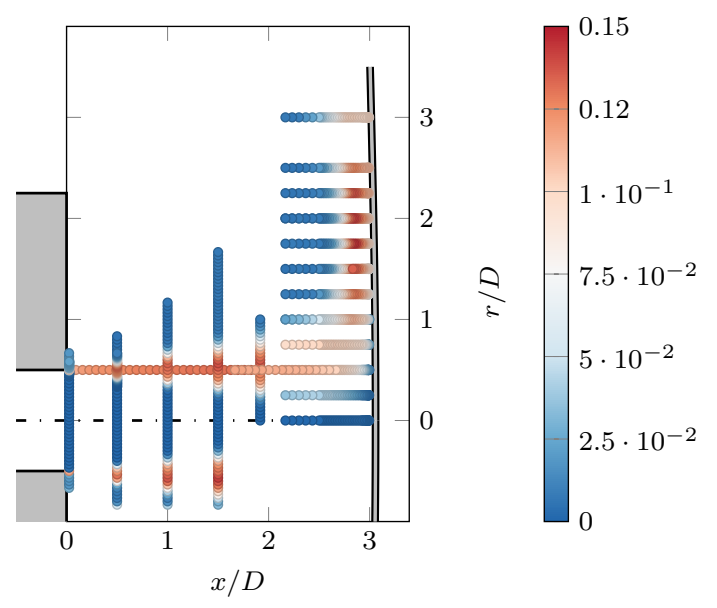

(b) $T^{\mathrm{RMS}} /\left(T_{j}-T_{e}\right)$

Figure 7: Mean (a) and fluctuating (b) temperature at the CWT measurement points.

The stagnation region (2) is characterized by the damping of the axial velocity fluctuations $u_{x}^{\prime}$ down to the wall (Fig. $8 \mathrm{c}, r / D=0$ ) whereas the radial velocity fluctuations $u_{r}^{\prime}$ tend to increase in the near wall region due to the development of the inner layer (Fig. $8 \mathrm{~d}, r / D=0$ ). The azimuthal velocity fluctuations $u_{\theta}^{\mathrm{RMS}}$ exhibit a similar flow structure as the radial fluctuating velocity (Fig. 6e).

In the wall jet $(\boldsymbol{B})$, the profiles of radial velocity fluctuations $u_{r}^{\mathrm{RMS}}$ at $r / D=1.5$ and 2 (Fig. $8 \mathrm{c}$ ) feature two distinct peaks, visible on the LDV profiles. The highest one is located in the outer layer at $\simeq 0.1 D$ above the wall while the other one appears in the close vicinity of the wall. The position of the local minimum between these peaks can be associated with the boundary between the inner layer and outer layer of the wall jet. This minimum disappears for higher radial locations. The highest radial velocity fluctuation $u_{r}^{\mathrm{RMS}}$ appears between $1.5<r / D<2$ and reaches $\sim 20 \%$ (Fig. $6 \mathrm{~d}$ ). In this region, the other fluctuating velocity components also reach their highest values, in the decreasing order $u_{r}^{\mathrm{RMS}}>u_{\theta}^{\mathrm{RMS}}>u_{x}^{\mathrm{RMS}}$ (Figs. 6c and 6e).

For radial locations $r / D \geq 1.5$, the profiles of temperature fluctuations in the wall jet exhibit a sudden change of slope (Fig. 8e, dotted red line) the position of which corresponds to the local minimum in radial velocity fluctuation, as highlighted by vertical black solid lines at $r / D=2$ in Fig. 8. The slope break might then be linked to the boundary between the inner and outer layers of the wall jet.

\subsection{Heat transfer}

Fig. 9 shows the Nusselt number $\mathrm{Nu}_{D}=h D / k_{f}$ and effectiveness $\eta=\left(T_{a w}-T_{e}\right) /\left(T_{j}-T_{e}\right)$ distribution along the plate (solid red line). These radial distributions result from azimuthal averaging of both $\mathrm{Nu}_{D}(y, z)$ and $T_{a w}(y, z)$ fields obtained by means of the ThEFA method presented in AppendixA. The min/max envelope (red fill) of the measured data is also plotted. Variations against the mean value are low for both $\mathrm{Nu}_{D}$ and $T_{a w}$ that confirms the excellent flow axisymmetry. Data from previous experiments are also represented, which are based on the thin foil technique applied on impinging jet configurations with similar parameters (table 2).

Table 2: Configuration parameters of previous experiments.

\begin{tabular}{lcccc} 
Authors & $\operatorname{Re}_{D}$ & $H / D$ & nozzle & \\
\hline \hline $\begin{array}{l}\text { Buchlin and } \\
\text { Laperches }\end{array}$ & 60000 & 2.6 & convergent & \\
$\begin{array}{l}\text { (1998) } \\
\text { Hofmann }\end{array}$ & 68000 & 2.5 & convergent & $\diamond$ \\
et al. (2007) & & & fully dev. pipe & $\odot-$ \\
Brevet (2001) & 23000 & 3 & convergent & $\square \square$ \\
Buchlin & 20000 & 3 & &
\end{tabular}

(2011)

Fig. 9a shows that the highest Nusselt number is reached at the stagnation point while a secondary maximum is observed at the radial location $r / D=2.1$. A secondary maximum was also observed at the same location by Hofmann et al. (2007) and at $r / D=2.5$ by Buchlin and Laperches (1998). However, the Nusselt number distribution significantly differs from the previous experiments in the stagnation region. The origin of this difference certainly lies in the jet exit condition. Contrary to the previous experiments, which used convergent nozzles, our jet issues a fully developed pipe. Several studies (e.g Roux et al., 2011) evidenced that the heat transfer coefficient at the stagnation point is higher for jet issuing a fully developed pipe than for those originating from a convergent nozzle, which exhibit low turbulence intensity and a flat velocity profile at the outlet section. Kataoka (1990) attributed the stagnation heat transfer enhancement to the turbulence already present in the jet core.

Fig. 9b shows that the measured effectiveness (red line) is close to unity in the stagnation region $(0<r / D<1)$ 
(a) $\left\langle u_{r}\right\rangle / U_{j}$

(b) $\left(\langle T\rangle-T_{e}\right) /\left(T_{j}-T_{e}\right)$
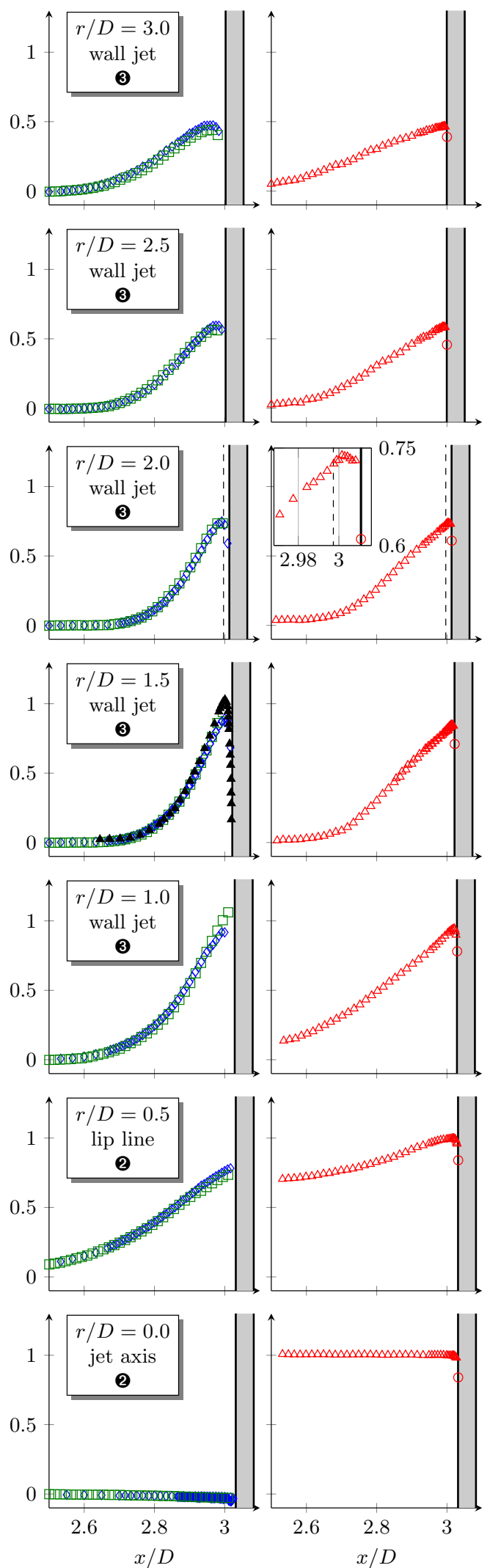

(c) $u_{r}^{\mathrm{RMS}} / U_{j}$

(d) $u_{x}^{\mathrm{RMS}} / U_{j}$

(e) $T^{\mathrm{RMS}} /\left(T_{j}-T_{e}\right)$
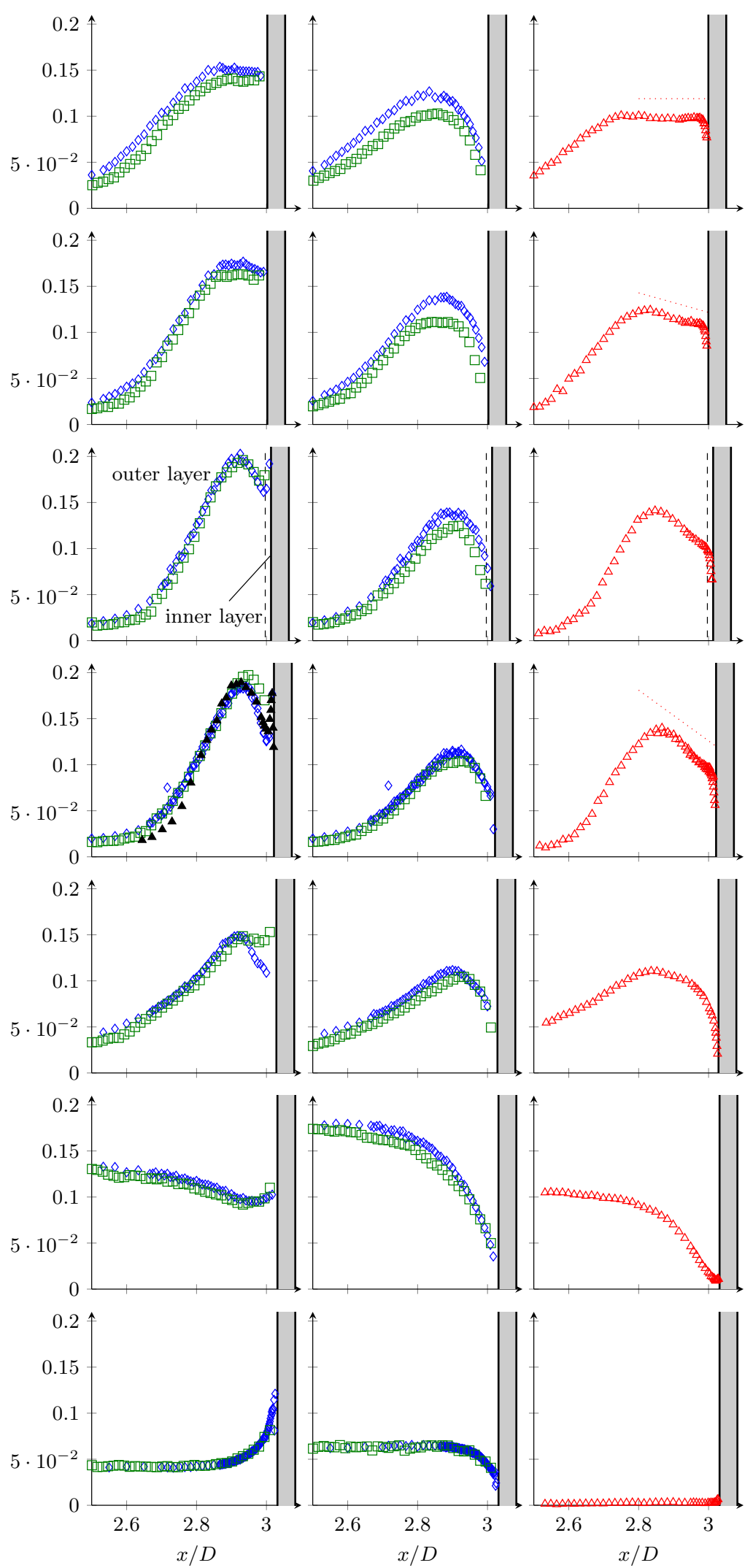

Figure 8: Profiles at different radial locations along the impinged plate. From left to right : (a) mean radial velocity, (b) mean temperature, (c) fluctuating radial velocity, (d) fluctuating axial velocity, (e) fluctuating temperature. ( $\square$ ) S-PIV, ( $\diamond)$ LDV, ( $\Delta$ ) CWT, (O) wall temperature (IRTh), (ム) experimental data of (Cooper et al., 1993) $\left(\operatorname{Re}_{D}=70,000, H / D=3\right.$, fully developed pipe flow) 


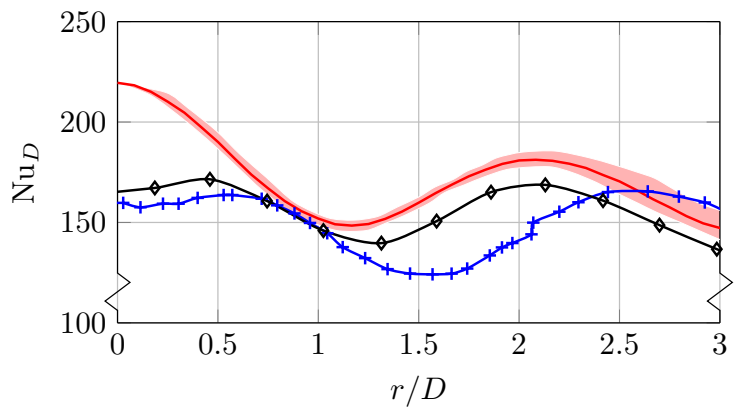

(a) Radial distribution of the mean Nusselt number

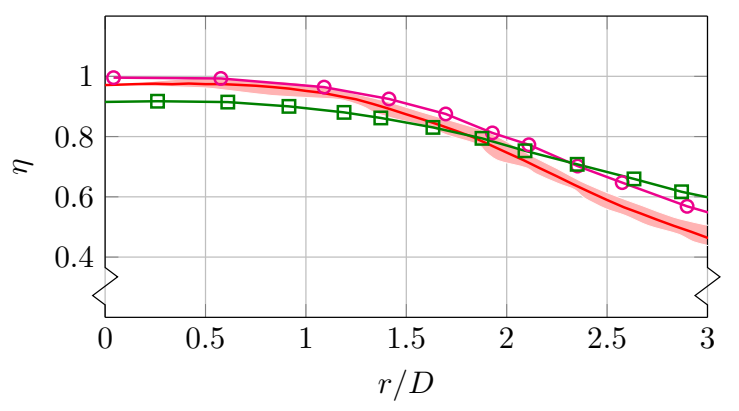

(b) Radial distribution of the effectiveness

Figure 9: Comparison of the (a) Nusselt number and (b) effectiveness on the targeted surface measured by the ThEFA ( $\_$) to previous experiments for similar configurations. Line legend is provided in table 2.

i.e. the adiabatic wall temperature $T_{a w}$ is close to the jet exit temperature $T_{j}$. This means that the stagnation region is only exposed to hot flow at temperature $T_{j}$, which has not yet undergone mixing with the cold ambient. This confirms that the impinged plate is located before the end of the jet core. For higher radial locations $r / D>1$, the effectiveness decreases monotonically due to the mixing with ambient air. Similar distributions were observed in previous experiments of Buchlin (2011) and Brevet (2001), conducted on lower Reynolds numbers configurations. As yet mentioned in the introduction, effectiveness was proved little sensitive to the jet Reynolds number, which is confirmed by the present comparison to lower $\operatorname{Re}_{D}$ configurations.

\subsection{Vortex detection}

The two-dimensional instantaneous vector fields as measured by S-PIV can be used to get a better view of the flow topology through the detection of large coherent structures. The algorithm of Debien et al. (2016) was employed to quantify both their size and location. Contrary to classical vortex extraction algorithms, this approach does not require any arbitrary threshold to define a vortex region and only makes use of the vector field topology. The method relies on the concept of closed loops the tangent of which presents a constant angle to the vector field. As illustrated in Fig. 10, the boundary of a vortex region (red curve) is then defined as the closed loop, encircling a focus, which starts and ends at a saddle point (magenda dot).

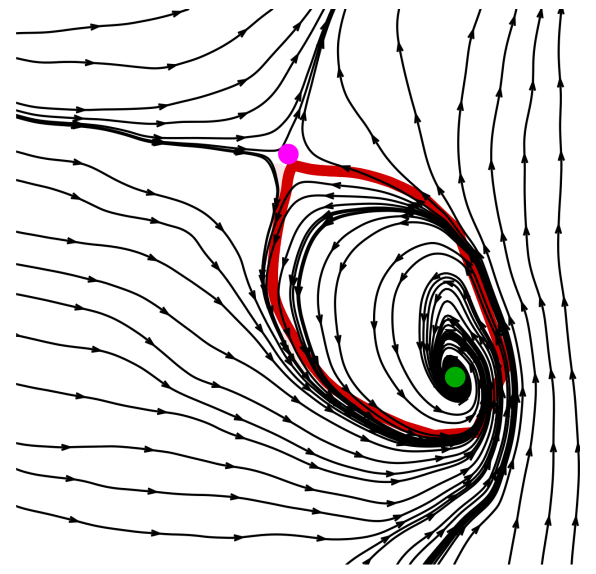

Figure 10: Principle of the vortex-region detection algorithm. Red line : boundary of a detected vortex region. Green dot : center of the vortex region (attracting focus). Magenta dot : saddle point.

The algorithm was applied on 5,000 snapshots, spatially filtered by a median filter in order to avoid detection of small eddies. Only the 1,500 largest detected vortex regions were retained. Their location and size are reported in Fig. 11. Insets show representative instantaneous velocity snapshots on which one or several vortex regions were detected. The mean path, along which the centers of the vortex-region cluster, is also represented.

The instantaneous streamlines plotted in inset (a) in Fig. 11 show small rolling-up turbulent structures downstream of the pipe exit. They are linked to the natural Kelvin-Helmholtz instability in the shear layer. The streamline pattern looks symmetrical with respect to the jet axis, that indicates the azimuthal coherence of ring-like vortices generated at the pipe exit.

The largest vortex regions were found in both the stagnation region and the wall jet [insets (b) and (c), Fig. 11]. Such vortical structures were evidenced in previous experiments (Popiel and Trass, 1991; Roux et al., 2011; Tummers et al., 2011) for lower Reynolds number jets. They are generally referred to as primary structures. Hadžiabdić and Hanjalić (2008) attributed these coherent structures to the remnants of the free-jet vortices. By means of smoke visualizations, Popiel and Trass (1991) pointed out that free-jet vortices are stretched when approaching the plate owing to the strong flow deviation, which finally increases their diameter. Downstream of impingement, the primary structure is convected along the wall. The mean path of the vortex-region centers (Fig. 11) suggests that these structures approach the most the plate between radial locations $r / D=1$ and $r / D=2$. In this range, the distance to the wall of the mean path is about $0.2 D$, which is similar to the findings of Roux et al. (2011) on a configuration $H / D=3$ and $\operatorname{Re}_{D}=28,000$.

\subsection{Spectral analysis}

In this section, the time-resolved velocity (LDV) and temperature (CWT) measurements are used to characterize the flow from a spectral point of view. 


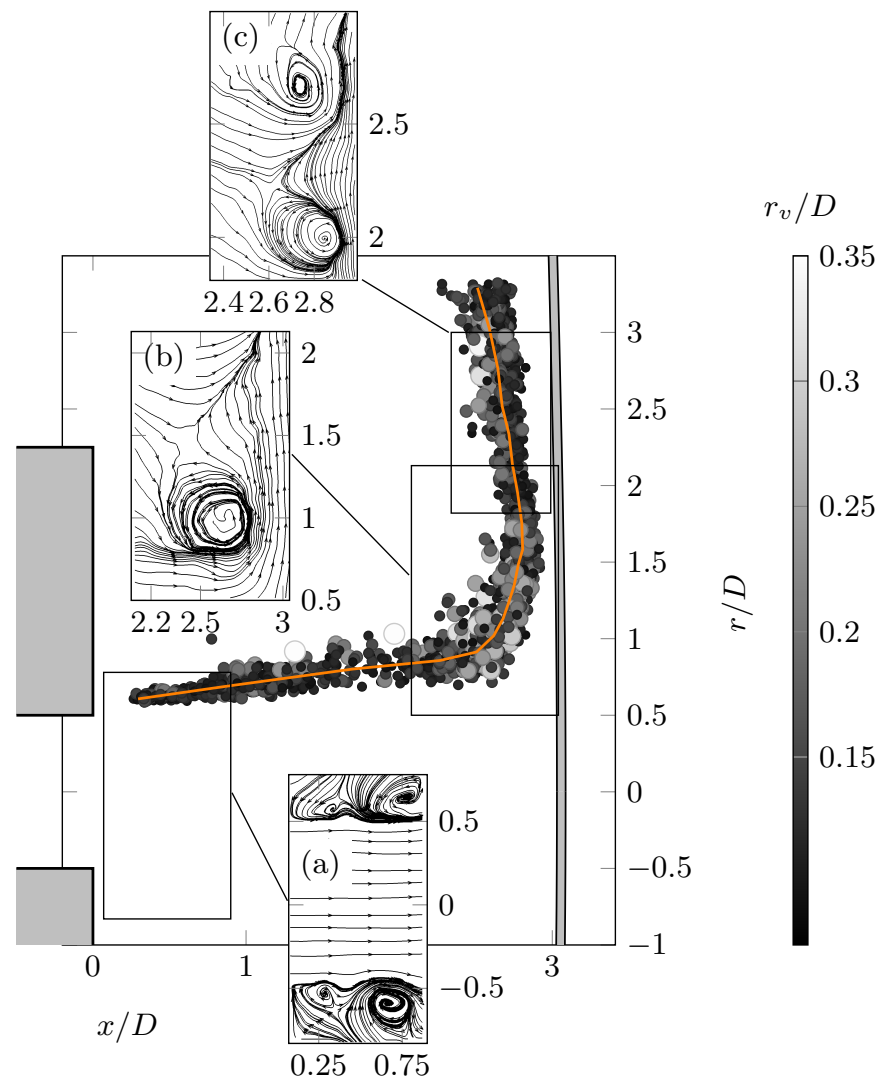

Figure 11: Location and size of the vortex regions. Grayscale represents the radius of the circle of equivalent area. Orange line corresponds to the mean path of the vortex-region centers.

Fig. 12 shows the $\|\cdot\|^{\infty}$-normalized power spectral density (PSD) of both radial velocity $u_{r}$ and temperature $T$ for a measurement point located in the shear layer of the free jet $(r / D=0.5$ and $x / D=2)$. On the logarithmic plot (Fig. 12a), the spectra features the classical $-5 / 3$ Kolmogorov law which corresponds to the inertial range of the turbulent energy cascade. The cut-off frequency associated with the thermal response of the cold-wire thermometer appears at lower frequency $(\simeq 3 \mathrm{kHz})$ compared to the velocity one.

A close-up in the low frequency range (Fig. 12b) shows the appearance of a peak around the frequency $f_{p}=$ $170 \mathrm{~Hz}$, which corresponds to a Strouhal number $\mathrm{St}_{D}=$ $f_{p} / U_{j} D=0.4$. This value lies in the typical range of Strouhal numbers that were observed in previous experiments (Crow and Champagne, 1971; O'Donovan and Murray, 2007) on both free and impinging jet configurations and is often referred to as the column mode of the jet.

From these observations, we derived a simple criterion (called $P$ in the following) in order to highlight the flow regions where the $170 \mathrm{~Hz}$-peak emerges in the PSD. The $P$-criterion is defined as the ratio between the mean PSD in the close vicinity of the peak $f_{p}$ and the mean PSD

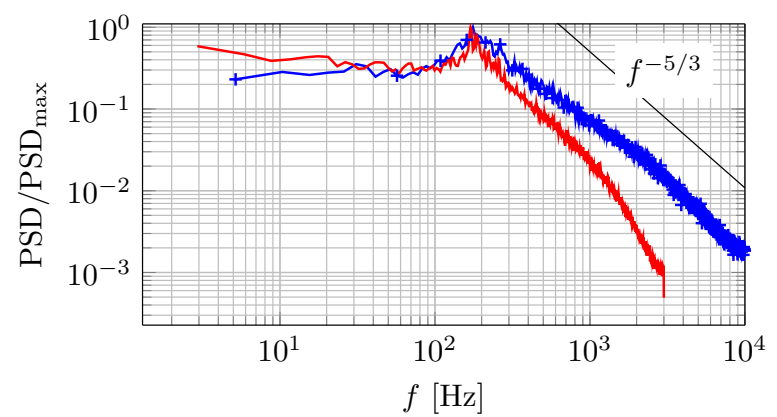

(a) Logarithmic plot for the whole spectra. Black solid line represents the $-5 / 3$ Kolmogorov law.

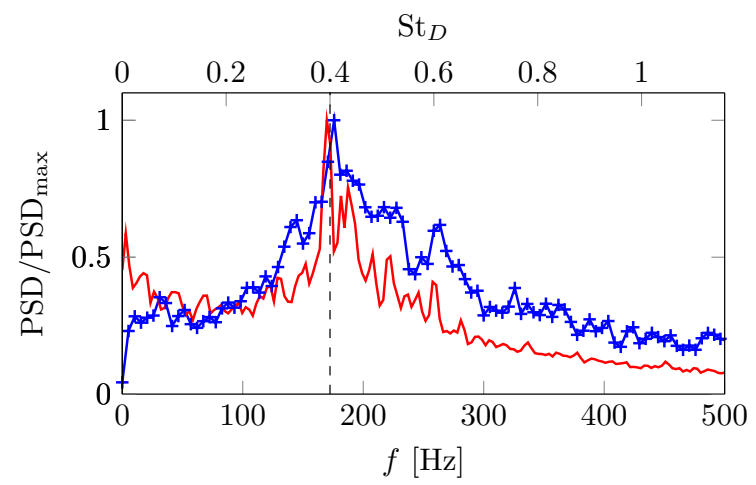

(b) Linear plot in the low frequency range.

Figure 12: Normalized PSD at $r / D=0.5$ and $x / D=2.0$. Frequency

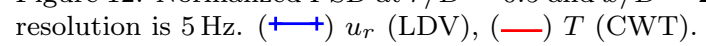

taken over a given range $\pm \Delta f$ around the peak:

$$
P\left(f_{p}\right)=\frac{\frac{1}{2 \Delta f_{p}} \int_{f_{p}-\Delta f_{p}}^{f_{p}+\Delta f_{p}} \operatorname{PSD}(f) \mathrm{d} f}{\frac{1}{2 \Delta f} \int_{f_{p}-\Delta f}^{f_{p}+\Delta f} \operatorname{PSD}(f) \mathrm{d} f}
$$

The averaging over the $\pm \Delta f_{p}$ range around $f_{p}$ was necessary to take into account the slight variability $( \pm 10 \mathrm{~Hz})$ of the actual frequency of the peak. Fig. 13 illustrates how the $P$-criterion applies on two representative PSD: the larger value the $P$-criterion takes, the more the $170 \mathrm{~Hz}$ frequency peak emerges. When no peak emerges, the $P$ criterion is close to unity. This approach was proven robust regarding the choice of $\Delta f$, at least to a certain extent. Considering the PSD shape, we selected $\Delta f=200 \mathrm{~Hz}$.

The application of the criterion on all measurement points of LDV and CWT is presented in Figs. 14, 15 and 16 for the axial $u_{x}$ and radial $u_{r}$ velocity components and temperature $T$, respectively. Representative PSD at particular measurement points are plotted on the right.

Fig. 15 shows that $\mathrm{St}_{D}=0.4$ is strongly marked in the jet core for the axial velocity $u_{x}$ [point (d), Fig. 15] while being almost absent along the $r / D=0.5$-line [point (c), Fig. 15]. These features can be recognized as the signature of the column mode which induces alternating acceleration-deceleration of the jet core velocity due to the most amplified coherent structures in the free jet 

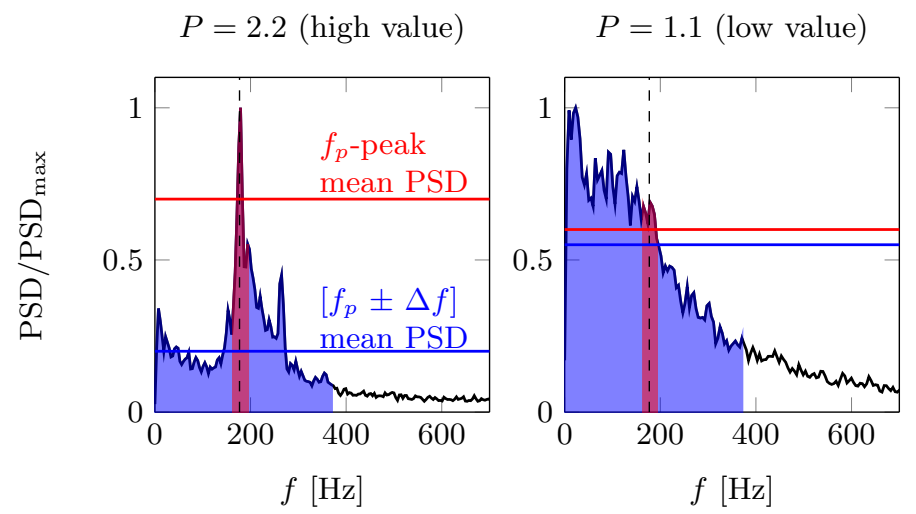

Figure 13: Calculation principle of the $P$-criterion for two representative PSD of $u_{x}$. Red area corresponds to the PSD in the $f_{p} \pm \Delta f_{p}$ range. Blue area corresponds to the PSD in the $f_{p} \pm \Delta f$ range.

shear layer (Crow and Champagne, 1971). In addition, it is shown that $\mathrm{St}_{D}=0.4$ appears from axial locations $x / D>1$ in the free-jet shear layer for the radial velocity $u_{r}$ [point (d), Fig. 15] and for the temperature $T$ [point (c), Fig. 16].

Interestingly, $\mathrm{St}_{D}=0.4$ still emerges in the stagnation region and the wall jet, down to the radial location $r / D=2.5$. Figs 14,15 and 16 show that the flow regions where the $P$-criterion reaches high levels are quite different depending on the quantity that is considered. The axial velocity $u_{x}$ (inset in Fig. 15) exhibits high level of $P$-criterion at $0.2 D$ above the wall [point (b), Fig. 15]. The $P$-criterion decreases when approaching the wall or moving away from the wall jet center. On the opposite, $P$-criterion for the radial velocity $u_{r}$ (inset in Fig. 16) features high levels in both the near-wall region [point (b), Fig. 16] and at $0.5 D$ above the wall [point (a), Fig. 16] whereas $\mathrm{St}_{D}=0.4$ does not emerge at the in-between position [point (c), Fig. 16]. Concerning temperature, $\mathrm{St}_{D}=0.4$ is only noticeable in the near-wall region [point (b), Fig. 16] while not being detected in the outer side of the wall jet.

This spectral analysis thus suggests that the free-jet instabilities observed at $\mathrm{St}_{D}=0.4$ drive the dynamics of the wall jet. The present conclusion is supported by the work of O'Donovan and Murray (2007), which showed that PSD of both velocity and heat transfer at different radial location along the plate exhibit a clear frequency peak at $\mathrm{St}_{D}=0.6$, corresponding to the free-jet instabilities of their configuration. They also noticed the strong coherence between the axial velocity $u_{x}$ and the wall heat transfer at this particular frequency. In the present study, we showed that the frequency associated with our column mode at $\mathrm{St}_{D}=0.4$ strongly emerges from PSD of $u_{x}, u_{r}$ and $T$ in distinct regions of the wall jet.

\subsection{Vortical dynamics and heat transfer}

The mean path of the primary structures as determined in Fig. 11 is represented in Figs. 14, 15 and 16. In the wall jet, this mean path goes through the flow region where $\mathrm{St}_{D}=0.4$ emerges for the axial velocity $u_{x}$ with high value of the $P$-criterion (inset in Fig. 15). On the opposite, the inset in Fig. 16 indicates that $\mathrm{St}_{D}=0.4$ for the radial velocity $u_{r}$ emerges on both sides of the mean path. Inset in Fig. 16 shows that the $170 \mathrm{~Hz}$-frequency for temperature $T$ emerges only between the mean path of the primary structures and the wall.

The $P$-criterion distribution around the mean path of the primary vortices can be explained if we assume that the passing frequency of the primary structures educed in Sec. 3.5 is the column-mode frequency $\mathrm{St}_{D}=0.4$. In this scope, we examine the flow field topology associated with a primary structure. In Fig. 17, an instantaneous velocity field from S-PIV measurements is used to illustrate how a primary vortex influences the flow field (The snapshot corresponds to the flow region where the primary structures were found to be the closest to the wall). One can see that the passage of a primary structure induces alternating positive and negative axial velocity fluctuations $u_{x}^{\prime}$. They are the highest at the level of the center of the primary structure, thus promoting the emergence of $\mathrm{St}_{D}=0.4$ for $u_{x}$ along the mean path.

The radial velocity $u_{r}$ intensity showed in Fig. 17 reveals that the primary structure is accompanied by an accelerating flow region on the wall side (right). On the left side (ambient), the whirling streamlines produce negative radial velocity. The radial fluctuations $u_{r}^{\prime}$ due to the passage of a primary structure are then located on each side of its center, which leads to energy content at $\mathrm{St}_{D}=0.4$ on each side of the mean path.

Even though no instantaneous temperature fields are available from the measurements, it can be yet thought that the primary structure is likely to bring cold air from the ambient side to the vicinity of the wall. From the viewpoint of a point located in this region, the passage of a primary structure therefore manifests itself as a temperature fluctuation $T^{\prime}$. Hence, $\mathrm{St}_{D}=0.4$ of $T$ emerges on the right side of the mean path. 
$\mathrm{PSD} / \mathrm{PSD}_{\max }$

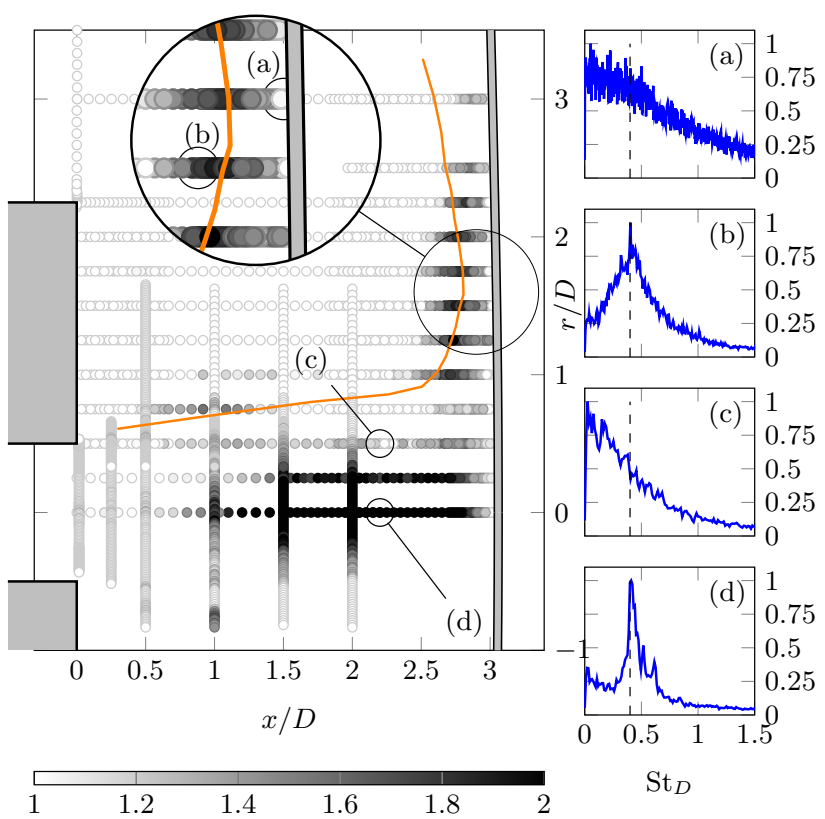

Figure 14: $P$-criterion for axial velocity $u_{x}$ at the LDV measurement points. Orange line : mean path of vortex regions. Left: PSD at particular locations. Dashed vertical lines represents $\mathrm{St}_{D}=0.4$.
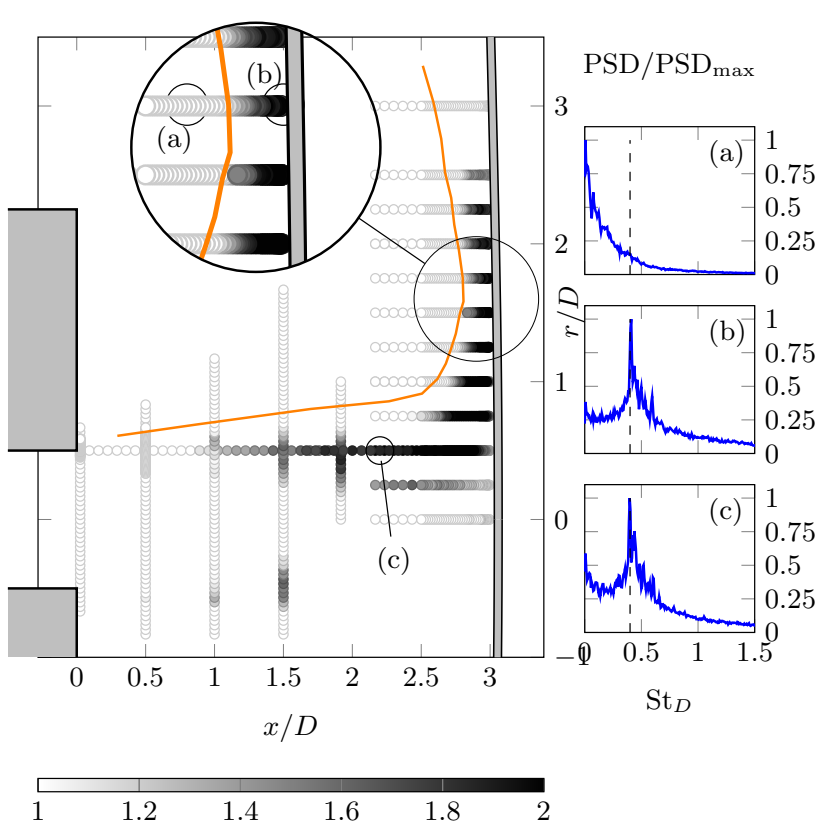

Figure 16: $P$-criterion for temperature $T$ at the CWT measurement points.

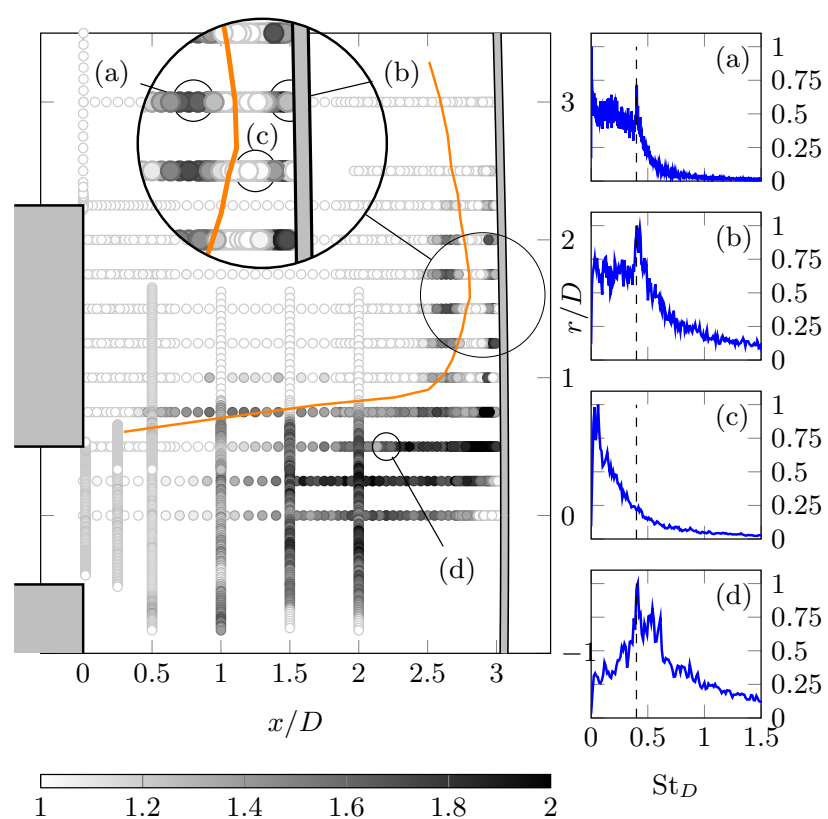

Figure 15: $P$-criterion for radial velocity $u_{r}$ at the LDV measurement points.
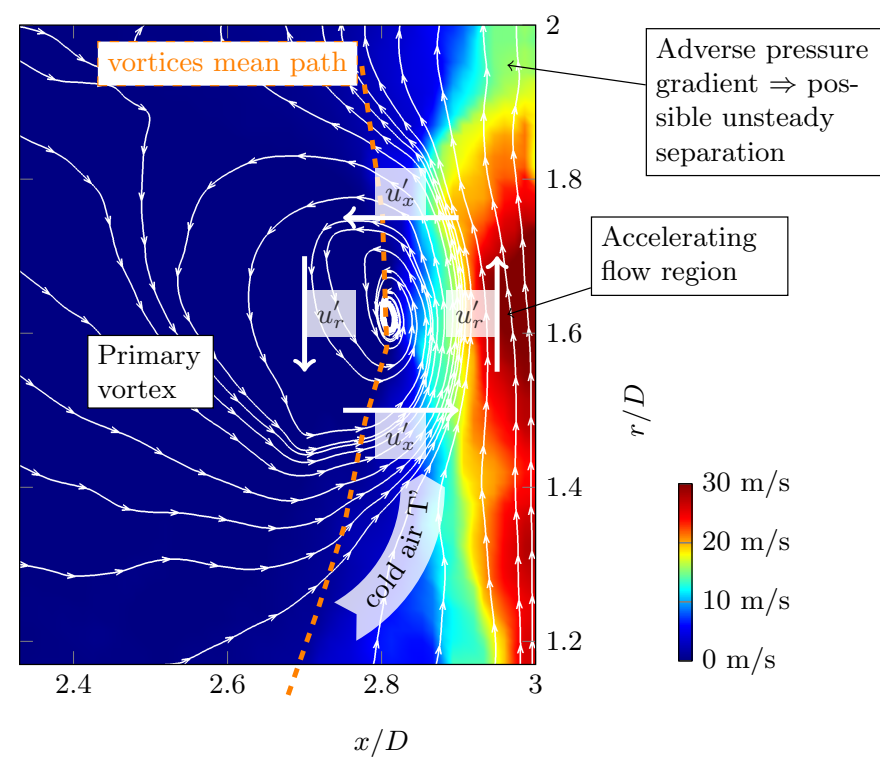

Figure 17: Instantaneous flow field around the radial location $r / D=1.6$ as observed on a S-PIV snapshot wherein a primary vortex is detected. Colormap represents the radial velocity $u_{r}$. 
Fig. 17 also gives an illustration of the deceleration undergone by the flow in the vicinity of the wall downstream of the primary vortex. This deceleration, if strong enough, can induce a localized near wall unsteady separation (often referred to as the secondary vortex) which moves along with the primary structure, as evidenced in several experiments for lower Reynolds numbers (Tummers et al., 2011). Unfortunately the spatial resolution of the present study did not permit to track down such small near-wall structures. It can be yet argued that, if unsteady separation occurs, it should most likely happen ahead of a large primary structure when it lies close to the wall. Such conditions are encountered in the region $1<r / D<2$ along the wall, as showed in Fig. 11. Furthermore, the ThEFA measurements showed that this region corresponds also to the increase of the Nusselt number from the minimum at $r / D=1.2$ to the secondary maximum at $r / D=2.1$ (Fig. 9a). These observations are consistent with the idea that unsteady separation plays a role in the apparition of secondary maximum in the Nusselt number distribution as discussed in several previous studies (Hadžiabdić and Hanjalić, 2008; Dairay et al., 2015).

\section{Conclusion}

In this work, various measurements techniques were applied to a hot impinging jet configuration at high Reynolds number and small nozzle-to-plate spacing, selected for its originality with respect to the literature. Both velocity and temperature fields were detailed, along with the Nusselt number and the adiabatic wall temperature distribution on the impinged plate. The results of the present study can be used as a valuable experimental database for validation of numerical simulations on similar configuration. The pipe exit condition was properly characterized through an analytical law for the mean velocity profile and the complete description of the Reynolds stress tensor. The good agreement between two different measurement techniques of velocity field enforces the relevance of the presented results. Moreover, the Nusselt number distribution, as obtained from an inverse conduction method, featured a secondary maximum as observed in previous experiments for small nozzle-toplate distances.

The spatially-resolved instantaneous velocity fields from S-PIV highlighted the presence of large turbulent structures known as primary structures. Based on a spectral analysis of the time-resolved measurements of both temperature and velocity, further evidence of coupled dynamics between free-jet instabilities and wall-jet turbulence at $\mathrm{St}_{D}=0.4$ were provided. Furthermore, a physical interpretation of the wall-jet velocity and temperature fluctuations at this Strouhal number was proposed based on S-PIV snapshots.
Finally, an analysis of the velocity field in the neighborhood of a primary structure evidenced the flow local acceleration near the wall and the subsequent deceleration downstream at a location where the secondary maximum in the Nusselt number distribution is observed. The present results thus support the idea proposed by several authors that the primary vortices are responsible of this secondary maximum through a possible unsteady separation in the decelerating flow region.

A comparison between numerical simulation results and the present experimental data will be presented in a forthcoming companion paper.

\section{Acknowledgments}

The present research was supported by Onera in the framework of a joint project about numerical and experimental investigation of jet configurations. The authors would like to thank Yves Le Sant for his assistance in the S-PIV calibration process and François Chedevergne for his insightfull advice. The help of Francis Micheli, Gillian Leplat, Jean-François Breil, Nicolas Fasano and Christian Pelissier in setting up the experimental rig is also acknowledged.

\section{AppendixA. ThEFA method for $\mathrm{Nu}_{D}$ and $T_{a w}$ de- termination}

The local convective heat transfer coefficient $h$ on the front face of the plate is defined by Newton's law:

$$
\begin{aligned}
q_{c}=q_{c}\left(T_{w}\right) & =h\left(T_{\text {ref. }}-T_{w}\right) \\
& =h\left(T_{a w}-T_{w}\right)
\end{aligned}
$$

As mentioned in the introduction, the reference temperature should be taken as the adiabatic wall temperature since the configuration is non-isothermal. The convective heat transfer coefficient is generally made dimensionless according to the pipe diameter $D$ to form the Nusselt number:

$$
\mathrm{Nu}_{D}=\frac{h D}{k_{f}\left(T_{f}\right)}
$$

where $k_{f}$ is the fluid conductivity evaluated at the film temperature $T_{f}=\left(T_{w}+T_{a w}\right) / 2$. The principle of calculation of $\mathrm{Nu}_{D}$ and $T_{a w}$ from rear face temperature measurements during the plate-heating transient is schematically described in Fig. A.18. The three main steps of the technique are as follows:

1. The plate, initially at uniform ambient temperature, is suddenly submitted to the jet flow. The infrared thermography then measures the transient evolution of the temperature on the rear face $T_{w}^{\mathrm{rear}}(y, z, t)$ until the thermal equilibrium is reached (Sec. 3.2) 


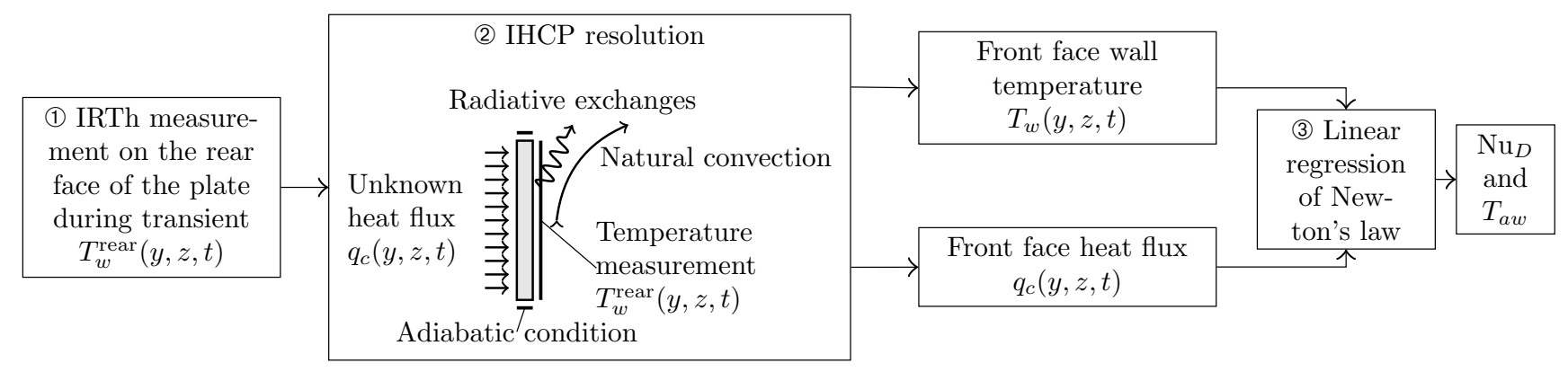

Figure A.18: Nusselt number $\mathrm{Nu}_{D}$ and adiabatic wall temperature $T_{a w}$ calculation by means the ThEFA method.

Table A.3: Thermal properties of the T40 titanium.

\begin{tabular}{ll}
\hline $\begin{array}{l}\text { Density } \rho_{p} \\
\text { Conduction }\end{array}$ & $4507 \mathrm{kgm}^{-3}$ \\
coefficient $k_{p}$ & $18.95-3.94 \times 10^{-3} T \mathrm{Wm}^{-1} \mathrm{~K}^{-1}$ \\
$\begin{array}{l}\text { Specific heat } \\
\text { capacity } C_{p}\end{array}$ & $348.2+0.429 T \mathrm{Jkg}^{-1} \mathrm{~K}^{-1}$ \\
\hline
\end{tabular}

2. The heat flux $q_{c}(y, z, t)$ on the front face is calculated from the rear face temperature measurement $T_{w}^{\text {rear }}(y, z, t)$ by means of resolution of an inverse heat conduction problem (IHCP), introduced by Reulet et al. (2003). The plate lateral sides are assumed adiabatic. The IHCP provides also the front face temperature $T_{w}(y, z, t)$ during the transient phase. At each time step $n$ of the transient, the unknown heat flux distribution on the plate $q_{c}^{n}$ to be estimated is obtained by minimizing the functional:

$$
R=\sum_{k=1}^{r} \sum_{i=1}^{N_{\text {meas. }}}\left(T_{i}^{\text {rear }, n+k}-T_{i}^{n+k}\left(q_{c}^{n}\right)\right)^{2}
$$

This functional represents the sum of the squared differences between the observations, i.e the rear face temperatures $T_{i}^{\text {rear }}$ measured by IRTh, and the computed temperature $T_{i}$ at the same location $i$ (direct problem). Following Beck's sequential approach (Beck et al., 1985), the heat flux distribution $q_{c}^{n}$ is assumed constant during $r$ future time steps. The plate temperature field calculation (direct problem) consists in the resolution of the unsteady threedimensionnal non-linear heat equation in the plate, that reads as follows:

$$
\rho_{p} C_{p} \frac{\partial T}{\partial t}=\nabla \cdot\left(k_{p} \nabla T\right)
$$

where the plate physical properties $\rho_{p}, C_{p}$ and $k_{p}$ depend explicitly on temperature (Table A.3). Table A.4 provides the parameters that were selected for the IHCP resolution. The number of grid points in the plate thickness direction $n_{x}$ is selected to properly capture the heat diffusion in the plate. The
Table A.4: Numerical parameters of the IHCP resolution.

\begin{tabular}{lc}
\hline Time step $\Delta t$ & $0.2 \mathrm{~s}$ \\
Number of future time steps $r$ & 5 \\
\hline Number of grid point in $O y$ direction & 96 \\
Number of grid point in $O z$ direction & 96 \\
Number of grid point in $O x$ direction & 3 \\
\hline
\end{tabular}

Fourier number is

$$
\text { Fo }=\frac{\alpha \Delta t}{\Delta x^{2}}=0.26 \quad \text { with } \quad \Delta x=e / n_{x}
$$

The choice of number of future time steps $r$ is based on the characteristic time interval required for a front-face heat flux change to influence the rear face temperature accordingly. Practically, it is chosen so that the "inverse Fourier number"

$$
\frac{\alpha r \Delta t}{e^{2}}
$$

is high enough ( $>0.07$ in our case) for stability reasons.

Heat flux boundary conditions need to be provided on the other faces. The local heat flux on the rear face $q_{c}^{\text {rear }}$ consists of both radiation and natural convection. It is computed from the surface radiative properties, through the Stefan-Boltzmann's law, and the heat convection coefficient using the following relation:

$$
q_{c}^{\text {rear }}=\varepsilon \sigma\left(T_{\text {rad }}^{4}-T_{w}^{4}\right)+h^{\text {rear }}\left(T_{e}-T_{w}\right)
$$

The radiative temperature $T_{\text {rad }}$ is taken equal to the ambient temperature $T_{e}$ while the emissivity $\varepsilon=0.82$ is uniform over the plate. The convection coefficient $h^{\text {rear }}$ is computed from an empirical correlation for natural convection over a vertical plate (Mac Adams, 1964):

$$
\begin{cases}\mathrm{Nu}_{L}=0.59 \mathrm{Ra}_{L}^{1 / 4} & \text { if } \mathrm{Ra}_{L}<10^{9} \\ \mathrm{Nu}_{L}=0.13 \mathrm{Ra}_{L}^{1 / 3} & \text { if } \mathrm{Ra}_{L}>10^{9}\end{cases}
$$

where $\mathrm{Nu}_{L}=h^{\text {rear }} L / k_{f}$ is the Nusselt number, based on the plate length $L$, and $\mathrm{Ra}_{L}=$ 


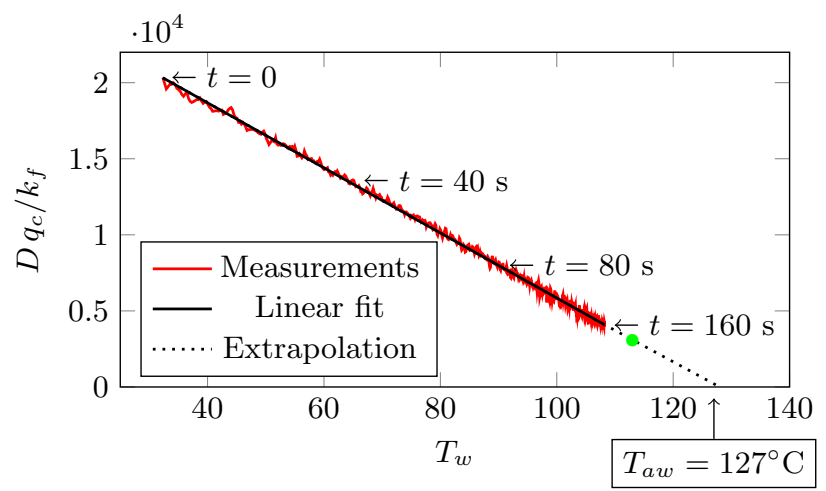

Figure A.19: Linear fit of the measured front face heat flux and wall temperature (from IHCP resolution) during the plate-heating transient at the stagnation point. The steady-state wall temperature at this point is $T_{w}=113^{\circ} \mathrm{C}(\bullet)$.

$\frac{\beta g}{\nu \alpha} L^{3}\left(\left\langle T_{w}\right\rangle_{y, z}-T_{e}\right)$ the Rayleigh number, where $\left\langle T_{w}(t)\right\rangle_{y, z}$ is the mean temperature of the plate at time $t$.

3. Once the front face temperature $T_{w}$ and heat flux $q_{c}$ are known everywhere on the plate at each time during the transient, both Nusselt number $\mathrm{Nu}_{D}$ and adiabatic wall temperature $T_{a w}$ are calculated through fitting of the relation (A.1). In order to take into account the variation of $k_{f}$ with temperature, linear regression is directly carried out on the following relation:

$$
\frac{D q_{c}}{k_{f}\left(T_{f}\right)}=\mathrm{Nu}_{D}\left(T_{w}-T_{a w}\right)
$$

where $D$ is the pipe diameter. Fig. A.19 shows the $D q_{c} / k_{f}$ term against the plate wall temperature $T_{w}$ during the plate-heating transient. After $160 \mathrm{~s}$, steady-state is almost reached. The linear regression slope corresponds to the Nusselt number $\mathrm{Nu}_{D}$ while the extrapolated $q_{c}=0$ abscissa indicates the adiabatic wall temperature $T_{a w}$. This approach is valid only if the linearity hypothesis of the relation (A.9) holds in the temperature range considered, which is the case in the present measurements as indicated in Fig. A.19. As adiabatic wall temperature is an extrapolated quantity, it is sensitive to the quality of the linear fit. At the stagnation point, uncertainty of its value is estimated at about $1^{\circ} \mathrm{C}$.

\section{References}

Baughn, J. W., Hechanova, A. E., Yan, X., Nov. 1991. An experimental study of entrainment effects on the heat transfer from a flat surface to a heated circular impinging jet. Journal of Heat Transfer 113 (4), 1023-1025.

Baughn, J. W., Shimizu, S., Nov. 1989. Heat transfer measurements from a surface with uniform heat flux and an impinging jet. Journal of Heat Transfer 111 (4), 1096-1098.
Beck, J. V., Blackwell, B., Clair, C. R. S., Oct. 1985. Inverse Heat Conduction: Ill-Posed Problems. James Beck.

Behnia, M., Parneix, S., Shabany, Y., Durbin, P. A., Feb. 1999. Numerical study of turbulent heat transfer in confined and unconfined impinging jets. International Journal of Heat and Fluid Flow 20 (1), 1-9.

Brevet, P., 2001. Etude expérimentale et numérique des transferts thermiques par impact de jet(s): application aux moteurs aéronautiques. Ph.D. thesis, Université de Poitiers.

Buchlin, J. M., 2011. Convective heat transfer in impinging- gasjet arrangements. Journal of Applied Fluid Mechanics 4 (2), 137149

Buchlin, J. M., Laperches, M., 1998. Detailed investigation of aerothermal behaviour of confined impinging jet. Quantitative Infrared Themography QIRT 98, 258-264.

Carlomagno, G. M., Ianiro, A., Oct. 2014. Thermo-fluid-dynamics of submerged jets impinging at short nozzle-to-plate distance: A review. Experimental Thermal and Fluid Science 58, 15-35.

Champagnat, F., Plyer, A., Besnerais, G., Leclaire, B., Davoust, S., Le Sant, Y., Mar. 2011. Fast and accurate PIV computation using highly parallel iterative correlation maximization. Experiments in Fluids 50 (4), 1169-1182.

Cooper, D., Jackson, D. C., Launder, B. E., Liao, G. X., Jul. 1993. Impinging jet studies for turbulence model assessment-I. Flowfield experiments. International Journal of Heat and Mass Transfer 36 (10), 2675-2684.

Crow, S. C., Champagne, F. H., 1971. Orderly structure in jet turbulence. Journal of Fluid Mechanics 48 (03), 547-591.

Dairay, T., Fortuné, V., Lamballais, E., Brizzi, L.-E., Feb. 2015. Direct numerical simulation of a turbulent jet impinging on a heated wall. Journal of Fluid Mechanics 764, 362-394.

Debien, A., Krbek, K. A. F. F. v., Mazellier, N., Duriez, T., Cordier, L., Noack, B. R., Abel, M. W., Kourta, A., Feb. 2016. Closedloop separation control over a sharp edge ramp using genetic programming. Experiments in Fluids 57 (3), 1-19.

Dewan, A., Dutta, R., Srinivasan, B., 2012. Recent trends in computation of turbulent jet-impingement impingement heat transfer. Heat Transfer Engineering 33 (4-5), 447-460.

Didden, N., Ho, C.-M., 1985. Unsteady separation in a boundary layer produced by an impinging jet. Journal of Fluid Mechanics $160(-1), 235$.

Gardon, R., Akfirat, J. C., Oct. 1965. The role of turbulence in determining the heat-transfer characteristics of impinging jets. International Journal of Heat and Mass Transfer 8 (10), 12611272 .

Geers, L., 2004. Multiple impinging jet arrays: an experimental study on flow and heat transfer. Ph.D. thesis, Technische Universiteit Delft.

Goldstein, R. J., Sobolik, K. A., Seol, W. S., Aug. 1990. Effect of entrainment on the heat transfer to a heated circular air jet impinging on a flat surface. Journal of Heat Transfer 112 (3), $608-611$.

Guerra, D. R. S., Su, J., Silva Freire, A. P., Jul. 2005. The near wall behavior of an impinging jet. International Journal of Heat and Mass Transfer 48 (14), 2829-2840.

Hadžiabdić, M., Hanjalić, K., 2008. Vortical structures and heat transfer in a round impinging jet. Journal of Fluid Mechanics $596,221-260$.

Hammad, K. J., Milanovic, I., 2011. Effect of reynolds number on the turbulent flow structure in the near-wall region of an impinging round jet. In: ASME-JSME-KSME 2011 Joint Fluids Engineering Conference. Vol. 1. pp. 2875-2882.

Hofmann, H. M., Kind, M., Martin, H., Sep. 2007. Measurements on steady state heat transfer and flow structure and new correlations for heat and mass transfer in submerged impinging jets. International Journal of Heat and Mass Transfer 50 (19-20), 3957-3965.

Hoogendoorn, C. J., Dec. 1977. The effect of turbulence on heat transfer at a stagnation point. International Journal of Heat and Mass Transfer 20 (12), 1333-1338. 
Jambunathan, K., Lai, E., Moss, M., Button, B., Jun. 1992. A review of heat transfer data for single circular jet impingement. International Journal of Heat and Fluid Flow 13 (2), 106-115.

Kataoka, K., 1990. Impingement heat transfer augmentation due to large scale eddies. International Heat Transfer Conference 9 1, 255-273.

Katti, V., Prabhu, S. V., Aug. 2008. Experimental study and theoretical analysis of local heat transfer distribution between smooth flat surface and impinging air jet from a circular straight pipe nozzle. International Journal of Heat and Mass Transfer 51 (1718), 4480-4495.

Landreth, C. C., Adrian, R. J., Jan. 1990. Impingement of a low Reynolds number turbulent circular jet onto a flat plate at normal incidence. Experiments in Fluids 9 (1-2), 74-84.

Lee, J., Lee, S., 1999. Stagnation region heat transfer of a turbulent axisymmetric jet impingement. Experimental Heat Transfer $12(2), 137-156$

Lytle, D., Webb, B. W., Aug. 1994. Air jet impingement heat transfer at low nozzle-plate spacings. International Journal of Heat and Mass Transfer 37 (12), 1687-1697.

Mac Adams, W. H., 1964. Transmission de chaleur.

Martin, H., 1977. Heat and mass transfer between impinging gas jets and solid surfaces. Advances in Heat Transfer 13, 1-60.

Meola, C., Cardone, G., Carmicino, C., Carlomagno, G., 2000. Fluid dynamics and heat transfer in an impinging air jet. In: 9th International Symposium on flow visualization, paper 429 .

Micheli, F., Lavieille, M., Millan, P., Sep. 2006. Assa, un outil de référence pour le traitement du signal en vélocimétrie laser. In: 10e Congrès Francophone de Techniques Laser (CFTL), Toulouse (France).

Nagib, H. M., Chauhan, K. A., Oct. 2008. Variations of von Karman coefficient in canonical flows. Physics of Fluids 20 (10), 101518.

Nishino, K., Samada, M., Kasuya, K., Torii, K., Jun. 1996. Turbulence statistics in the stagnation region of an axisymmetric impinging jet flow. International Journal of Heat and Fluid Flow 17 (3), 193-201.

O'Donovan, T. S., Murray, D. B., Aug. 2007. Jet impingement heat transfer - Part II: A temporal investigation of heat transfer and local fluid velocities. International Journal of Heat and Mass Transfer 50 (17-18), 3302-3314.

Popiel, C. O., Trass, O., May 1991. Visualization of a free and impinging round jet. Experimental Thermal Fluid Science 4, 253264.

Reulet, P., Nortershauser, D., Millan, P., Aug. 2003. Inverse method using infrared thermography for surface temperature and heat flux measurements. In: 20th International Congress on Instrumentation in Aerospace Simulation Facilities. pp. 118-126.

Roux, S., Fénot, M., Lalizel, G., Brizzi, L. E., Dorignac, E., Jul. 2011. Experimental investigation of the flow and heat transfer of an impinging jet under acoustic excitation. International Journal of Heat and Mass Transfer 54 (15-16), 3277-3290.

Sodjavi, K., Montagné, B., Bragan,ca, P., Meslem, A., Byrne, P., Degouet, C., Sobolik, V., 2016. PIV and electrodiffusion diagnostics of flow field, wall shear stress and mass transfer beneath three round submerged impinging jets. Experimental Thermal and Fluid Science 70, 417-436.

Striegl, S. A., Diller, T. E., 1984. The effect of entrainment temperature on jet impingement heat transfer. Journal of heat transfer 106 (1), 27-33.

Toonder, J. M. J. d., Nieuwstadt, F. T. M., Nov. 1997. Reynolds number effects in a turbulent pipe flow for low to moderate Re. Physics of Fluids 9 (11), 3398-3409.

Tummers, M. J., Jacobse, J., Voorbrood, S. G., Nov. 2011. Turbulent flow in the near field of a round impinging jet. International Journal of Heat and Mass Transfer 54 (23-24), 4939-4948.

Vinze, R., Chandel, S., Limaye, M. D., Prabhu, S. V., Jan. 2016. Influence of jet temperature and nozzle shape on the heat transfer distribution between a smooth plate and impinging air jets. International Journal of Thermal Sciences 99, 136-151.
Violato, D., Ianiro, A., Cardone, G., Scarano, F., Oct. 2012. Threedimensional vortex dynamics and convective heat transfer in circular and chevron impinging jets. International Journal of Heat and Fluid Flow 37, 22-36.

Viskanta, R., Feb. 1993. Heat transfer to impinging isothermal gas and flame jets. Experimental Thermal and Fluid Science 6 (2), $111-134$.

Wu, X., Moin, P., Aug. 2008. A direct numerical simulation study on the mean velocity characteristics in turbulent pipe flow 608 , 81-112

Zagarola, M. V., Smits, A. J., 1998. Mean-flow scaling of turbulent pipe flow. Journal of Fluid Mechanics 373, 33-79.

Zuckerman, N., Lior, N., 2006. Jet impingement heat transfer: Physics, correlations, and numerical modeling. Advances in Heat Transfer 39, 565-631. 